\title{
ARTICLE
}

Received 17 Jun 2014 | Accepted 14 Oct 2014 | Published 21 Nov $2014 \quad$ DOl: 10.1038/ncomms6566

\section{PKM2 phosphorylates MLC2 and regulates cytokinesis of tumour cells}

Yuhui Jiang ${ }^{1}$, Yugang Wang ${ }^{1}$, Ting Wang ${ }^{2}$, David H. Hawke ${ }^{3}$, Yanhua Zheng ${ }^{1}$, Xinjian Li ${ }^{1}$, Qin Zhou ${ }^{4}$, Sadhan Majumder ${ }^{5}$, Erfei Bi ${ }^{2}$, David X. Liu ${ }^{6}$, Suyun Huang ${ }^{7} \&$ Zhimin Lü,8,9

Pyruvate kinase M2 (PKM2) is expressed at high levels during embryonic development and tumour progression and is important for cell growth. However, it is not known whether it directly controls cell division. Here, we found that Aurora B phosphorylates PKM2, but not PKM1, at T45; this phosphorylation is required for PKM2's localization and interaction with myosin light chain 2 (MLC2) in the contractile ring region of mitotic cells during cytokinesis. PKM2 phosphorylates MLC2 at Y118, which primes the binding of ROCK2 to MLC2 and subsequent ROCK2-dependent MLC2 S15 phosphorylation. PKM2-regulated MLC2 phosphorylation, which is greatly enhanced by EGF stimulation or EGFRvIII, K-Ras G12V and B-Raf $\mathrm{V} 600$ E mutant expression, plays a pivotal role in cytokinesis, cell proliferation and brain tumour development. These findings underscore the instrumental function of PKM2 in oncogenic EGFR-, K-Ras- and B-Raf-regulated cytokinesis and tumorigenesis.

\footnotetext{
${ }^{1}$ Brain Tumor Center and Department of Neuro-Oncology, The University of Texas MD Anderson Cancer Center, Houston, Texas 77030, USA. ${ }^{2}$ Department of Cell and Developmental Biology, Perelman School of Medicine, University of Pennsylvania, Philadelphia, Pennsylvania 19104, USA. ${ }^{3}$ Department of Systems Biology, The University of Texas MD Anderson Cancer Center, Houston, Texas 77030, USA. ${ }^{4}$ The M.O.E. Key Laboratory of Laboratory Medical Diagnostics, College of Laboratory Medicine, Chongqing Medical University, Chongqing, 400016, China. ${ }^{5}$ Department of Genetics, The University of Texas MD Anderson Cancer Center, Houston, Texas 77030, USA. ${ }^{6}$ Department of Pharmaceutical Sciences, Washington State University College of Pharmacy, Spokane, WA 99202, USA. ${ }^{7}$ Departments of Neurosurgery, The University of Texas MD Anderson Cancer Center, Houston, Texas 77030, USA. ${ }^{8}$ Department of Molecular and Cellular Oncology, The University of Texas MD Anderson Cancer Center, Houston, Texas 77030, USA. ${ }^{9}$ Cancer Biology Program, The University of Texas Graduate School of Biomedical Sciences at Houston, Houston, Texas 77030, USA. Correspondence and requests for materials should be addressed to Z.L. (email: zhiminlu@mdanderson.org).
} 
C ytokinesis is the final stage of cell division, during which the two daughter cells separate completely ${ }^{1,2}$. A failure in cytokinesis completion can lead to polyploidy, a state in which cells contain more than two sets of chromosomes; this results in cell cycle arrest and cell growth inhibition ${ }^{3}$. Cellular myosin II, the conventional two-headed myosin that was first identified in muscle tissue, is the primary motor protein responsible for cytokinesis. Myosin II comprises a parallel dimer of heavy chains, each of which binds to an essential light chain and a regulatory myosin light chain (RMLC) ${ }^{4}$. RMLC has smooth muscle isoforms (MRLC1 or MLC-2C, encoded by $M Y L 9$ ), non-muscle isoforms (encoded by the highly homologous MYL12A and MYL12B, referred to as MYLB12A and MYLB12B hereafter) and cardiac ventricular muscle isoform (MLC2 or MLC2v, encoded by MYL2). Phosphorylation of MRLC1 and MYLB12A/B at Ser19 (or Thr18/Ser19) in higher eukaryotes and phosphorylation of human MLC2 at Ser15 allow myosin II to interact with actin, assemble an actomyosin complex and initiate contraction $^{5-7}$. Thus, RMLC phosphorylation controls both the assembly and contractility of the actomyosin contractile apparatus $^{8}$.

Pyruvate kinase regulates the final rate-limiting step of glycolysis, which catalyses the transfer of a phosphate group from phosphoenolpyruvate (PEP) to adenosine diphosphate for the production of pyruvate and adenosine triphosphate (ATP $)^{9,10}$. Alternate splicing of PKM pre-mRNA by hnRNP $\mathrm{A} 1 / 2$ and polypyrimidine tract binding (PTB) protein splicing factors leads to PKM2 generation by the inclusion of exon 10 and the exclusion of exon 9, which is specific for PKM1 (refs 11,12). Besides its cytosolic roles in glycolysis ${ }^{13-18}$, PKM2, which is upregulated by growth factor receptor activation and phosphorylated by extracellular signal-regulated kinase, translocates into the nucleus and binds to c-Src-phosphorylated Y333 of $\beta$-catenin, which is essential for $\beta$-catenin transactivation ${ }^{19-22}$. Histone H3-interacted PKM2 phosphorylates histone $\mathrm{H} 3$ at T11, leading to $\mathrm{H} 3-\mathrm{K} 9$ acetylation and gene transcription, including $M Y C$ and CCND1 (encoding for cyclin D1) ${ }^{23,24}$. c-Myc expression results in the upregulation of GLUT1, lactate dehydrogenase A, and in a positive feedback loop, PTB-dependent PKM2 expression, which leads to an enhanced Warburg effect ${ }^{21}$. Cyclin D1 expression, in turn, promotes G1-S phase transition $19,23,25$. In addition to its specific role in regulating G1-S transition, PKM2 binds to and phosphorylates the spindle checkpoint protein Bub3, thereby regulating correct kinetochoremicrotubule attachment, the spindle-assembly checkpoint and accurate chromosome segregation ${ }^{26}$. However, whether PKM2 plays a role in other phases of the cell cycle besides the G1-S phase transition and mitotic checkpoint is not known.
In this study, we found that Aurora B phosphorylates PKM2 at T45 and that this phosphorylation is required for PKM2 to localize in the contractile ring of dividing cells, where it binds to MLC2 and phosphorylates MLC2 Y118. MLC2 Y118 phosphorylation primes ROCK2-mediated MLC2 S15 phosphorylation and is required for oncogenic protein-regulated cytokinesis progression.

\section{Results}

PKM2 Interacts with MLC2 and Is Critical for Cytokinesis. To determine whether PKM2 has functions in cytokinesis, we immunostained U87 human glioblastoma (GBM) cells and found that PKM2 was localized in the contractile ring or cleavage furrow as well as in the equator region of a large percentage of dividing cells (Fig. 1a, Supplementary Fig. 1a). Localization of PKM2 in these regions was also observed in HeLa cervical cancer cells and U87 cells expressing active epidermal growth factor receptor (EGFR) vIII mutant (Supplementary Fig. 1a), which lacks 267 amino acids from the extracellular domain of EGFR and is commonly found in GBM as well as in the breast, ovarian, prostate, and lung carcinomas ${ }^{27}$.

To avoid the effect of PKM2 depletion on G1-S transition and chromosome segregation and determine whether PKM2 regulates cytokinesis, we expressed doxycycline-inducible PKM2 shRNA in U87/EGFRvIII cells. These cells were synchronized from a double-thymidine block and release and arrested at metaphase by MG132 treatment; this was followed by removal of MG132 for cytokinesis $^{28}$. As demonstrated in Fig. 1b, PKM2 was largely depleted at the time that MG132 was removed. About $10-12 \%$ cells could not recover from arrested metaphase because of the toxic effect of MG132, and the rest of the cells underwent cell division. Live cell analyses revealed that a large percentage of U87/EGFRvIII cells with PKM2 depletion failed to complete cytokinesis and cell division, with regression of the cleavage furrow (Fig. 1c and Supplementary Fig. 1b). PKM2 depletioninduced retarded cleavage furrow formation and failure of cell division were also observed by immunofluorescence (IF) analyses (Supplementary Fig. 1c). Some cells exhibited a chromosome segregation defect that resulted from incomplete cell synchronization and PKM2 depletion in early mitosis that affected correct kinetochore-microtubule attachment ${ }^{26}$. These results indicate that PKM2 is involved in regulating tumour cell cytokinesis.

Myosin II, including its component RMLC, directly regulates contractile ring progression during cytokinesis. Quantitative PCR analyses of several human GBM cell lines and mouse embryonic fibroblasts (MEFs) showed expression of MLC2, MYL9 and

Figure 1 | PKM2 interacts with MLC2 and is critical for cytokinesis. Immunoblotting (b,d-f,h) and immunofluorescence (a,c,g,i) analyses were performed with the indicated antibodies. Nuclei were stained with DAPI (blue). (a) U87 cells in cytokinesis were immunostained with the indicated antibodies. Scale bars, $10 \mu \mathrm{m}$. (b) U87/EGFRvIll cells, synchronized by thymidine double block ( $2 \mathrm{mM}$ ), were released for the indicated time periods. Doxycycline $\left(500 \mathrm{ng} \mathrm{ml}^{-1}\right)$ was added at the indicated time point to induce PKM2 shRNA expression. MG132 $(25 \mu \mathrm{M})$ was added at the indicated time point to sustain the cells in metaphase for $6 \mathrm{~h}$. (c) U87/EGFRvIll cells expressing mCherry-histone H2B (for chromosome staining) were synchronized by thymidine double block. PKM2 shRNA was induced by doxycycline, as described in (b). MG132 was removed after $6 \mathrm{~h}$ incubation, followed by imaging analyses using a DeltaVision deconvolution microscope with a $\times 20$ lens in a $\mathrm{CO}_{2}$ environment chamber. Selected time points are shown. Scale bars, $10 \mu \mathrm{m}$. (d) The indicated cells were treated with or without EGF $\left(100 \mathrm{ng} \mathrm{ml}^{-1}\right)$ for $24 \mathrm{~h}$. (e,f) U87 cells (e) or U87 cells expressing Flag-PKM2 (f), which had been synchronized by double thymidine block $(2 \mathrm{mM})$ for $43 \mathrm{~h}$, were unreleased or released for $9 \mathrm{~h}$, followed by MG132 (25 $\mu \mathrm{M})$ treatment for $1.5 \mathrm{~h}$ to arrest cells at metaphase. MG132 was removed for 30 min before cell harvesting. Immunoprecipitates with the indicated antibodies were incubated with or without CIP (10 units) for $30 \mathrm{~min}$ at $37^{\circ} \mathrm{C}$ and were washed with PBS three times. C, cytokinesis; I, interphase (no thymidine release). (g) Flag-MLC2-expressing U87 cells, synchronized by double thymidine block $(2 \mathrm{mM})$ for $43 \mathrm{~h}$, were released for $10 \mathrm{~h}$ (anaphase). Scale bars, $10 \mu \mathrm{m}$. (h) Control or MLC2 shRNA was expressed in U87/EGFRvIll cells. (i) U87/EGFRvIll cells expressing MLC2 shRNA and mCherry-histone H2B were synchronized. MG132 (25 $\mu$ M) was added at the indicated time point as shown in (b) and incubated with the cells for $1.5 \mathrm{~h}$ to sustain the cells in metaphase. MG132 were then removed, followed by imaging analyses using a DeltaVision deconvolution microscope. Selected time points are shown. Scale bars, $10 \mu \mathrm{m}$. 
MYL12A/B (Supplementary Fig. 1d). Intriguingly, expression of EGFRvIII mutant significantly enhanced $M L C 2$ mRNA levels in U87 GBM cells (Supplementary Fig. 1d). Immunoblotting analyses showed that EGF treatment and EGFRvIII expression led to greatly increased MLC2 protein levels (Fig. 1d). These results indicate that EGFR activation, which greatly enhances PKM2 expression ${ }^{22}$, also upregulates MLC2 expression in tumour cells.
The specific contractile ring localization of PKM2 suggests that it interacts with RMLC, which is a regulatory component of myosin II. To test this assumption, we synchronized U87 cells with a double thymidine block and release and MG132 treatment. We next immunoblotted immunoprecipitated MLC2 or MRLC1 and MYLB12A/B with antibodies for PKM2 and PKM1. We found that PKM2 rather than PKM1 bound to endogenous MLC2 (Fig. 1e, left panels) but not MRLC1 and MYLB12A/B (Fig. 1e,

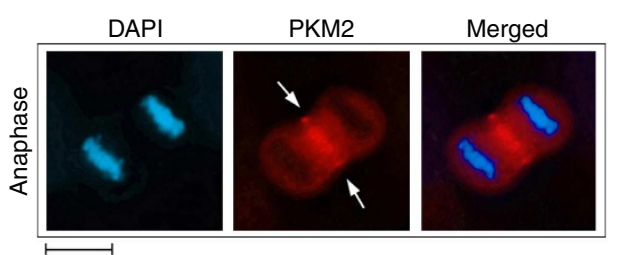

b

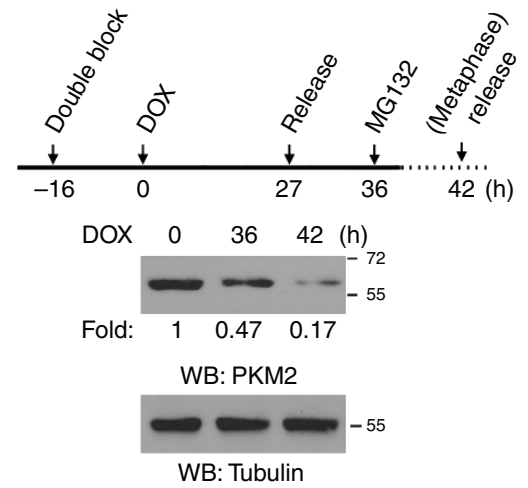

C
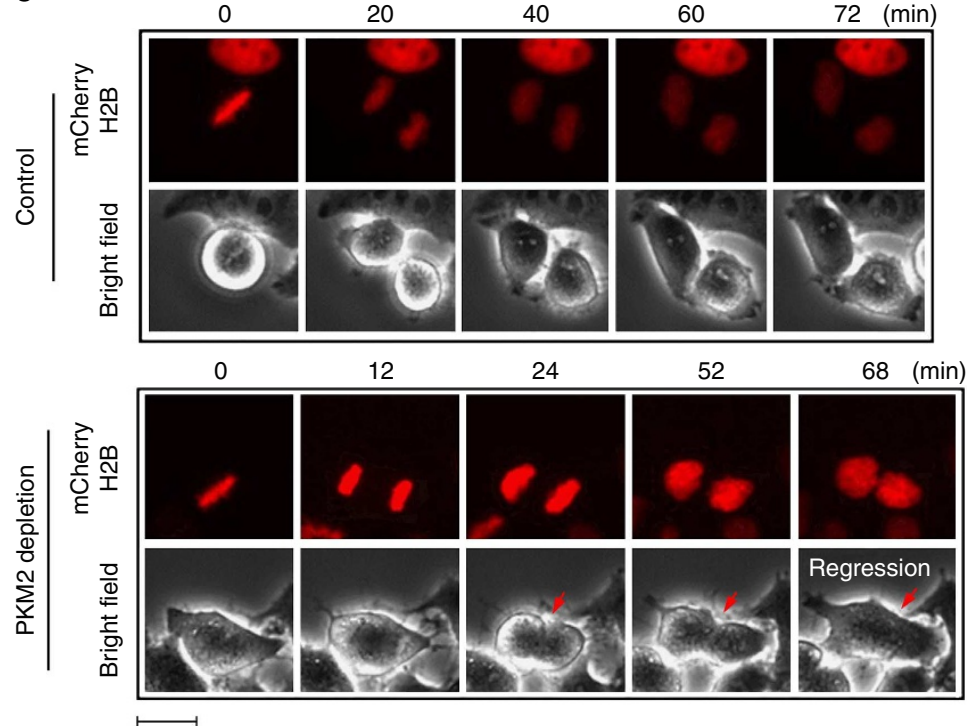

d

e
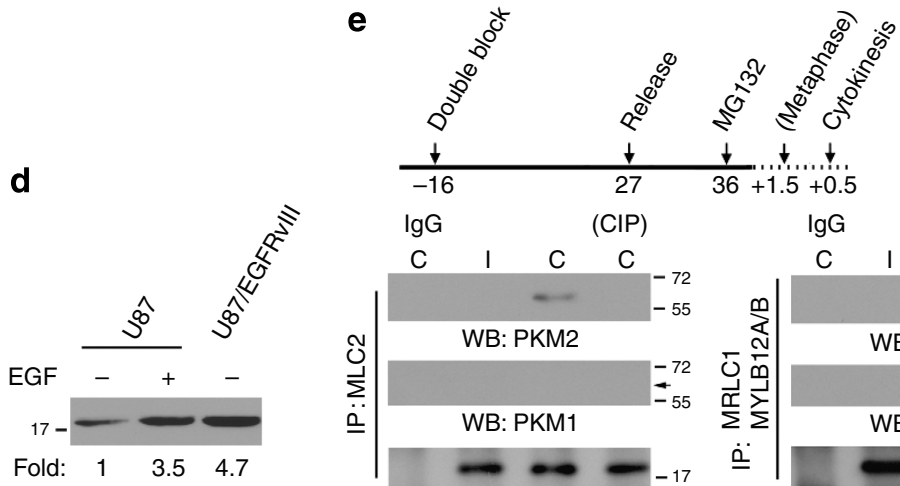

WB: MLC2

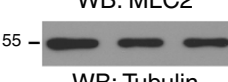

WB: Tubulin

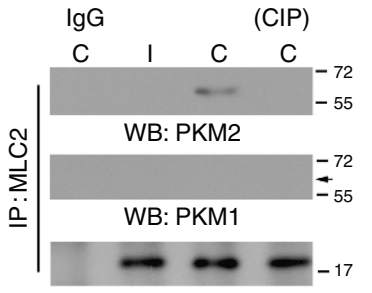

WB: MLC2

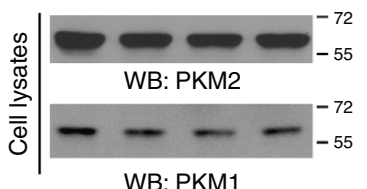

$\lg G$

$(\mathrm{CIP})$

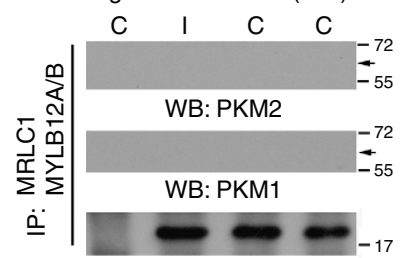

WB: MRLC1,MYLB12A/B

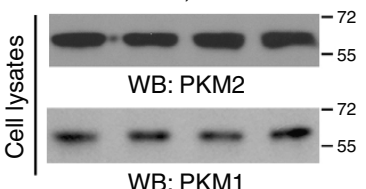

f
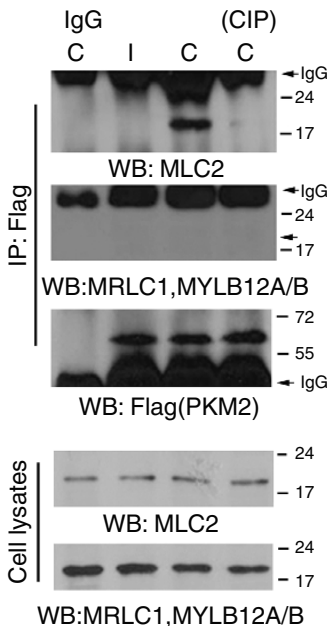

g Anaphase

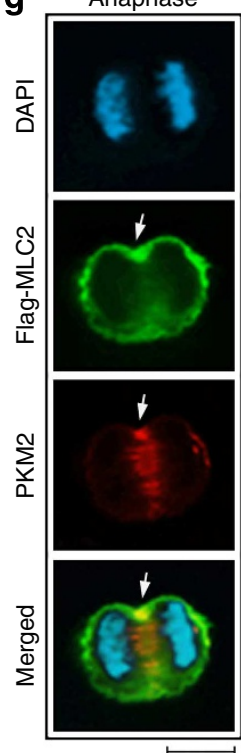

h

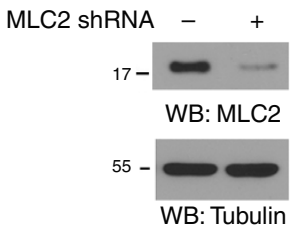

i

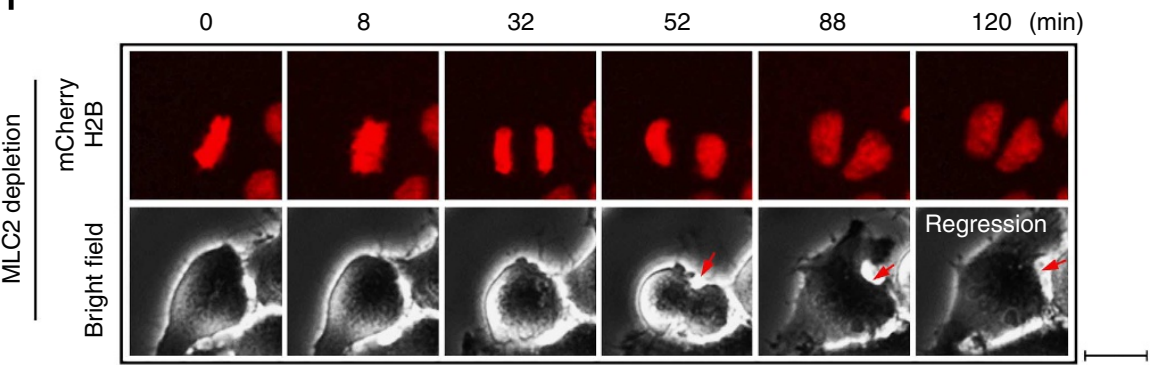


right panels) in U87 cells in cytokinesis, but not in interphase. This finding was further confirmed by reciprocal immunoprecipitation of Flag-tagged PKM2 in U87 (Fig. 1f) and U87/EGFRvIII (Supplementary Fig. 1e) cells and immunoblotting analyses with an anti-MLC2 antibody and an antibody for MRLC1 and MYLB12A/B. In addition, IF analysis indicates PKM2 partially co-localized with MLC2 in the contractile ring region (Fig. $1 \mathrm{~g}$ and Supplementary Fig. 1f). Intriguingly, incubating the precipitates with calf intestinal phosphatase (CIP) abrogated the interaction between PKM2 and MLC2 (Fig. 1e,f, and Supplementary Fig. 1e), and this abrogated effect was blocked by including phosphatase inhibitors (sodium orthovanadate, sodium pyrophosphate and sodium fluoride) (Supplementary Fig. 1e). These results suggest that protein phosphorylation is required for the binding of PKM2 to MLC2. Live cell analyses revealed that the expression of MLC2 shRNA in U87/EGFRvIII cells (Fig. 1h) blocked cytokinesis and cell division, with regression of the cleavage furrow, in a large percentage of the cells (Fig. 1i and Supplementary Fig. 1g). These results indicate that PKM2 interacts with MLC2 in a phosphorylation-dependent manner and that both PKM2 and MLC2 play a critical role in tumour cell cytokinesis.
Aurora B-phosphorylated PKM2 T45 Interacts with MLC2. To determine whether PKM2 is phosphorylated during cytokinesis and whether the phosphorylation of PKM2 is required for its binding to MLC2, we conducted liquid chromatography-coupled ion trap mass spectrometry (LC-MS/MS) analyses of tryptic digests of PKM2 in the MLC2 immunoprecipitates and found that PKM2 was phosphorylated at T45 (Fig. 2a).

Several Ser/Thr protein kinases, including Aurora B, Rhoassociated protein kinase (ROCK) and MLC kinase (MLCK), are known to be involved in cytokinesis ${ }^{2,29}$. Co-immunoprecipitation analyses revealed that PKM2 was associated only with Aurora B in cytokinesis but not in the interphase of U87 cells (Fig. 2b), and PKM2 largely co-localized with Aurora B in the spindle midzone of the cells (Supplementary Fig. 2a). In addition, this association was enhanced in U87/EGFRvIII cells (Supplementary Fig. 2b). We performed an in vitro kinase assay in which purified recombinant glutathione $S$-transferase (GST)-Aurora B was incubated with His-PKM2 proteins; Aurora B phosphorylated wild-type (WT) PKM2, but not PKM2 T45A, which was detected by both autoradiography and a specific anti-phospho-PKM2 T45 antibody (Fig. $2 c$ and Supplementary Fig. 2c). Treating U87 cells
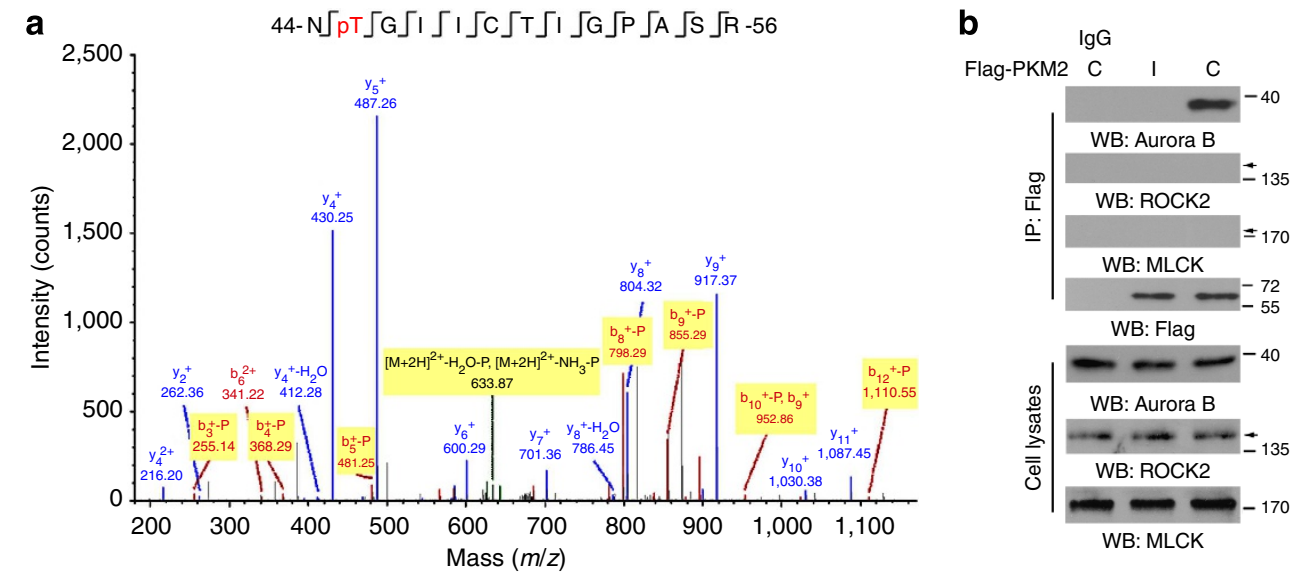

C
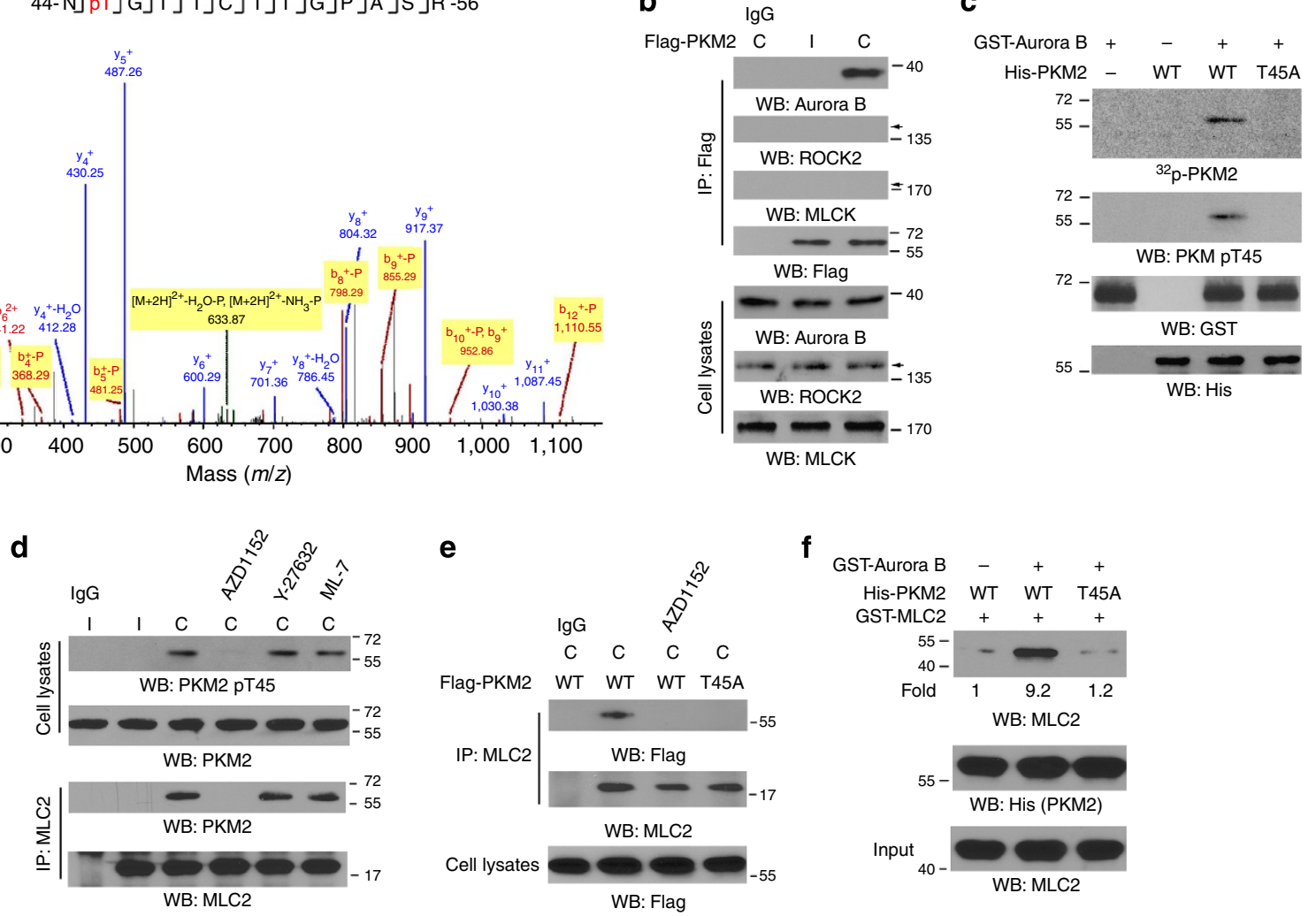

Figure 2 | Aurora B-phosphorylated PKM2 T45 interacts with MLC2. (a) PKM2, which was in the immunoprecipitate with an anti-MLC2 antibody from U87 cells in cytokinesis, was analysed by mass spectrometry. The results of a fragment spectrometrum of a tryptic peptide at $\mathrm{m} / z 691.827$ (mass error, - 2.39 p.p.m.) matched the doubly charged peptide 44-NTGIICTIGPASR-56, suggesting that T45 was phosphorylated. The Sequest score for this match was Xcorr $=3.19$. The Mascot score was 46, and the expectation value was 5.1e-4. (b) U87 cells that had been synchronized by double thymidine block $(2 \mathrm{mM})$ for $43 \mathrm{~h}$ were unreleased or released for $10 \mathrm{~h}$. Immunoprecipitation analyses with the indicated antibodies were performed. $\mathrm{C}$, cytokinesis; I, interphase (no thymidine release). (c) In vitro kinase assays were performed by mixing purified recombinant GST-Aurora B, His-PKM2 proteins and 32P-ATP. (d) U87 cells that had been synchronized by double thymidine block $(2 \mathrm{mM})$ for $43 \mathrm{~h}$ were unreleased or released for $9 \mathrm{~h}$, followed by MG132 $(25 \mu \mathrm{M})$ treatment for $1.5 \mathrm{~h}$ to arrest cells at metaphase. MG132 was removed, and the cells were treated with or without AZD1152 (100 nM), Y-27632 $(10 \mu \mathrm{M})$ or ML-7 $(10 \mu \mathrm{M})$ for 30 min before cell harvesting. (e) U87 cells that had been synchronized by double thymidine block (2 mM) for $43 \mathrm{~h}$ were released for $9 \mathrm{~h}$, followed by MG132 $(25 \mu \mathrm{M})$ treatment for $1.5 \mathrm{~h}$. MG132 was removed, and the cells were treated with or without AZD1152 $(100 \mathrm{nM})$ for 30 min before cell harvesting. Immunoprecipitation with the indicated antibodies was performed. (f) WT His-PKM2 or His-PKM2 T45A on agarose beads was incubated with or without purified Aurora B protein for a kinase assay; it was then washed in PBS three times and incubated with recombinant GST-MLC2. 
with AZD1152 Aurora B, Y-27632 ROCK and ML-7 MLCK inhibitors (inhibiting MRLC1 S19 phosphorylation) inhibited their respective kinase activity (Supplementary Fig. 2d), revealing that phosphorylation of WT PKM2 and the interaction between PKM2 and MLC2 in U87 cells in cytokinesis were inhibited only by AZD1152 (Fig. 2d). In contrast to its Flag-tagged WT counterpart, PKM2 T45A mutant largely lost its ability to interact with MLC2 (Fig. 2e).

To further evaluate the role of Aurora B in the interaction between PKM2 and MLC2, we mixed immobilized recombinant WT His-PKM2 or His-PKM2 T45A on agarose beads with recombinant GST-MLC2, with or without purified Aurora B protein for PKM2 phosphorylation. Figure $2 \mathrm{f}$ shows that Aurora $\mathrm{B}$ enhanced the binding of WT PKM2, but not PKM2 T45A mutant, to MLC2. In addition, PKM1, unlike PKM2, was unable to bind to MLC2 (Supplementary Fig. 2e). These results indicate that Aurora B phosphorylates PKM2 at T45, leading to an enhanced interaction between PKM2 and MLC2 during cytokinesis.

Mutations of the polar or hydrophobic surface residues of R400, L401, P403, L404, D407, P408, T409, and K433, coded by the PKM2-specific exon 10, and the expression of these mutants showed that the K433E mutant, but not others, reduced the binding ability of PKM2 to Aurora B (Supplementary Fig. 2f) and was resistant to be phosphorylated at T45 during cytokinesis (Supplementary Fig. 2g). PKM2 K433 can bind Tyrphosphorylated proteins ${ }^{19,30}$. Incubating the PKM2 precipitates with calf intestinal phosphatase abrogated the interaction between
PKM2 and Aurora B (Supplementary Fig. 2h), suggesting that a phosphorylation event is involved in the formation of the complex. In addition, IF studies showed that Flag-PKM2 K433E was unable to translocate to the contractile ring or co-localize with MLC2 (Supplementary Fig. 2i) during cytokinesis. These results indicate that PKM2 K433 is involved in binding to Aurora $\mathrm{B}$ and is required for translocation of PKM2 to the contractile ring region during cytokinesis.

PKM2 Phosphorylates MLC2 at Y118. PKM2 can act as a dualspecificity protein kinase that phosphorylates both the Ser/Thr and Tyr residues of its substrates ${ }^{23,31}$. To determine whether MLC2 is a substrate of PKM2 kinase activity, we performed an in vitro kinase assay by mixing recombinant Aurora B and MLC2 with WT PKM2, PKM2 K367M inactive mutant, or PKM1. Figure 3a shows that PKM2, but not PKM2 K367M or PKM1, phosphorylated MLC2; MLC2 phosphorylation was detected by autoradiography using ${ }^{32} \mathrm{P}$-labelled PEP (the physiological phosphate group donor of PKM2) and anti-phospho-Tyr, but not anti-phospho-Ser or anti-phospho-Thr antibodies. Of note, this phosphorylation did not occur in the presence of ATP, the physiological phosphate group donor of classic protein kinases. An LC-MS/MS analysis of MLC2 phosphorylated by PKM2 showed that MLC2 is phosphorylated at Y118 (Fig. 3b). Mutations of Y118 and Y152, which are the only two Tyr residues in MLC2, into phenylalanine revealed that only MLC2 Y118F, not WT MLC2 or MLC2 Y152F, was resistant to

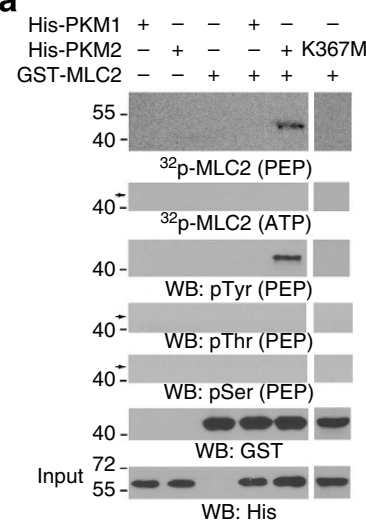

e

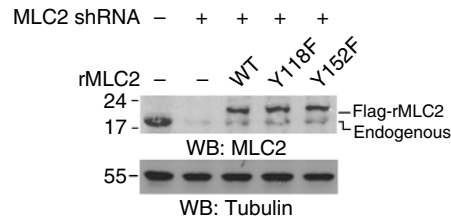

b

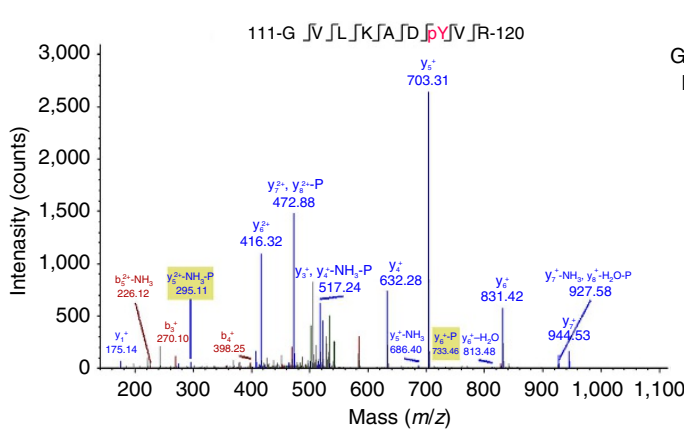

c

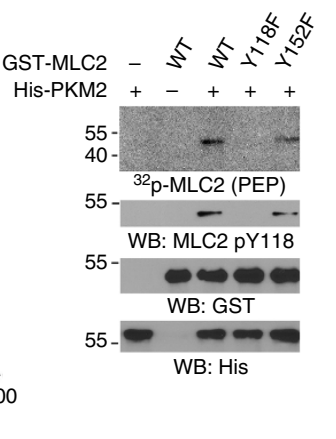

d

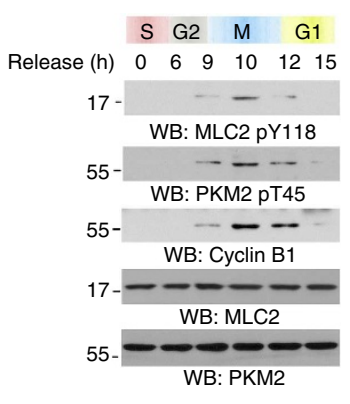

Figure 3 | PKM2 Phosphorylates MLC2 at Y118. (a,c-g), Immunoblotting analyses were performed with the indicated antibodies. C, cytokinesis;

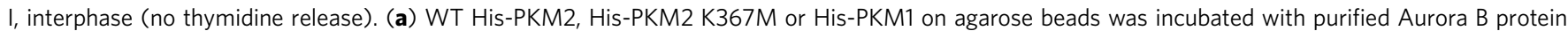
for a kinase assay; it was then washed in PBS three times and incubated with recombinant GST-MLC2 to proceed the kinase assay in the presence of

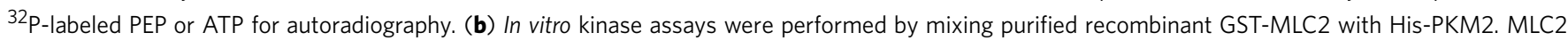
was analysed by mass spectrometry. The results of a mass spectrometric analysis of a tryptic fragment at $\mathrm{m} / \mathrm{z} 550.779$ (mass error, -0.16 p.p.m.) matched those of the doubly charged peptide 111-GVLKADYVR-120, suggesting that Y118 was phosphorylated. The Sequest score for this match was $X$ corr $=2.83$. The Mascot score was 51, and the expectation value was 1.1 e -4 . (c) In vitro kinase assays were performed by mixing the indicated

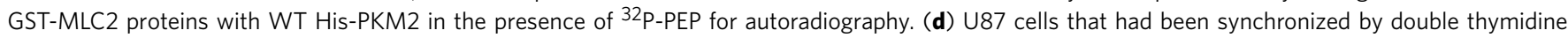
block $(2 \mathrm{mM})$ for $43 \mathrm{~h}$ were released for the indicated time periods. (e) U87 cells, with and without MLC2 shRNA expression, were reconstituted with or without expression of WT rMLC2, rMLC2 Y118F or rMLC2 Y152F (left panel). The cells were synchronized by double thymidine block (2 mM) for $43 \mathrm{~h}$

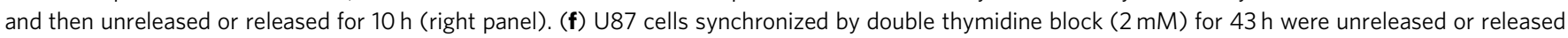
for $9 \mathrm{~h}$, followed by MG132 $(25 \mu \mathrm{M})$ treatment for $1.5 \mathrm{~h}$ to arrest cells at metaphase. MG132 was replaced with AZD1152 (100 nM), Y-27632 (10 $\mu$ M) or ML-7 $(10 \mu \mathrm{M})$ for 30 min before cell harvesting. (g) U87 cells with PKM2 depletion were stably transfected with a vector expressing WT rPKM2 or rPKM2 T45A. These cells were synchronized by double thymidine block $(2 \mathrm{mM})$ for $43 \mathrm{~h}$ and then unreleased or released for $10 \mathrm{~h}$. 
PKM2-mediated phosphorylation, as detected by autoradiography and an antibody that specifically recognizes phosphorylated MLC2 Y118 (Fig. 3c and Supplementary Fig. 3a). In addition, PKM2 T45A, which largely reduced its binding to MLC2, inefficiently phosphorylated MLC2 Y118 (Supplementary Fig. 3b). These results indicate that Aurora B phosphorylated PKM2 phosphorylates MLC2 Y118 in vitro.

Synchronized U87 (Fig. 3d) and HeLa (Supplementary Fig. 3c) cells with a double thymidine block and release had prominent MLC2 Y118 and PKM2 T45 phosphorylations at late-stage mitosis/cytokinesis (as indicated by cyclin B1 expression levels, which started to decrease at the beginning of anaphase ${ }^{32}$ ), and these phosphorylations were decreased in the G1 phase of the cell cycle. In addition, depletion of MLC2 with MLC2 shRNA and reconstituted expression of RNAi-resistant (r)MLC2 WT, rMLC2 Y118F, or rMLC2 Y152F in U87 (Fig. 3e, left panel) and U251 (Supplementary Fig. 3d) GBM cells revealed that rMLC2 Y118F, but not WT rMLC2 and rMLC2 Y152F, was resistant to the enhanced phosphorylation during cytokinesis of U87 (Fig. 3e, right panel) and U251 (Supplementary Fig. 3e) cells. IF studies showed that MLC2 Y118F and Y152F mutations did not affect MLC2 localization during cytokinesis (Supplementary Fig. 3f), suggesting that the resistance of MLC2 Y118F to phosphorylation was not due to its mis-localization.

Consistent with the role of Aurora B in the interaction between PKM2 and MLC2, AZD1152 Aurora B inhibitor, but not Y-27632 ROCK or ML-7 MLCK inhibitor, blocked PKM2-dependent phosphorylation of MLC2 at Y118 in cytokinesis (Fig. 3f) and in the contractile ring region of mitotic cells (Supplementary Fig. 3g). Furthermore, PKM2 depletion with PKM2 shRNA and reconstituted WT rPKM2 or rPKM2 T45A expression in U87 (Supplementary Fig. 3h, left panel) or U251 cells (Supplementary Fig. 3h, right panel) revealed that rPKM2 T45A expression abrogated MLC2 Y118 phosphorylation in the cytokinesis of U87 (Fig. 3g) and U251 (Supplementary Fig. 3i) cells and the contractile ring region of dividing cells (Supplementary Fig. 3j). Thus, these results indicate that Aurora B-dependent PKM2 phosphorylation at T45 is required for PKM2-dependent MLC2 phosphorylation at Y118 during cytokinesis.

MLC2 pY118 Primes ROCK2-mediated MLC2 pS15. Human MLC2 phosphorylation at S15 is known to be critical for actomyosin contraction $6,33,34$. To determine whether MLC2 Y118 phosphorylation has a regulatory effect on MLC2 S15 phosphorylation, we performed immunoblotting and IF studies with a specific MLC2 pS15 antibody (Supplementary Fig. 4a); these studies revealed that MLC2 S15 phosphorylation primarily occurred in cytokinesis, not in the interphase (Fig. 4a, left panel). In addition, this phosphorylation was restricted in the contractile ring region in U87 cells with reconstituted expression of WT rMLC2, but not rMLC2 Y118F, in the presence of MLC2 shRNA expression (Fig. 4a, middle and right panels, single cell; Supplementary Fig. 4b, multiple cells). In contrast, rMLC2 Y118F expression did not affect S19 phosphorylation of MRLC1 and MYLB12A/B (Supplementary Fig. 4c). Furthermore, depletion of endogenous MLC2 and reconstituted expression of WT rMLC2 or rMLC2 S15A in U87 cells revealed that the MLC2 S15A mutation did not affect MLC2 phosphorylation at Y118 (Supplementary Fig. 4d). These results indicate that MLC2 Y118 phosphorylation primes MLC2 S15 phosphorylation.

Depletion of endogenous PKM2 and reconstituted rPKM2 T45A expression in U87 cells (Supplementary Fig. 3h) revealed inhibited MLC2 S15 phosphorylation in cytokinesis and in the contractile ring region of dividing cells (Fig. 4b; Supplementary Fig. 4e, multiple cells). These results indicate that Aurora
B-dependent PKM2 T45 phosphorylation, which regulates MLC2 Y118 phosphorylation, is required for MLC2 S15 phosphorylation. The evidence that MLC2 S15A was still detected in the contractile ring region (Supplementary Fig. 4f) and that MLC2 S15A and Y118F mutants still interacted with PKM2 during cytokinesis (Supplementary Fig. 4g) further support that Aurora B-dependent PKM2 T45 phosphorylation is required for the interaction between PKM2 and MLC2.

AMP-activated protein kinase $(\mathrm{AMPK})^{35,36}, \mathrm{MLCK}^{37}$ and ROCK $2^{38}$ have been found to directly or indirectly regulate the phosphorylation of different RMLC isoforms. We treated U87 cells with Y-27632 ROCK inhibitor, compound C AMPK inhibitor (blocking downstream acetyl-CoA carboxylase (ACC) phosphorylation) and ML-7 MLCK inhibitor, which inhibited their respective kinase activities (Supplementary Figs $2 \mathrm{~d}$ and $4 \mathrm{~h}$ ); MLC2 S15 phosphorylation was primarily blocked by ROCK inhibition (Fig. 4c). ROCK1 and ROCK2 are known to be involved in cytokinesis ${ }^{29,39}$. Co-immunoprecipitation analyses revealed that MLC2 interacted with ROCK2, but not ROCK1 or AMPK, in cytokinesis (Fig. 4d). In addition, an in vitro kinase assay with mixed recombinant GST-ROCK2 and MLC2 revealed that ROCK2 phosphorylated WT MLC2, but not MLC2 S15A mutant, indicating that it phosphorylates MLC2 at S15 in vitro (Fig. 4e).

We found that Aurora B-dependent PKM2 T45 phosphorylation is required for PKM2-dependent MLC2 Y118 phosphorylation, which primes ROCK2-dependent MLC2 S15 phosphorylation. In line with these findings, we showed that Aurora B inhibition with AZD1152 and reconstituted expression of rPKM2 T45A (Fig. 4f, left panel) or rMLC2 Y118F (Fig. 4f, right panel) mutants, which abrogated MLC2 Y118 phosphorylation, blocked the interaction between MLC2 and ROCK2 and the phosphorylation of MLC2 at S15. The requirement of PKM2dependent MLC2 phosphorylation for the interaction between MLC2 and ROCK2 was further supported by the results of a GST-MLC2 pull-down assay. GST-MLC2 but not GST-MLC2 Y118F mutant interacted with ROCK2 in the presence of PKM2 and PEP (Fig. 4g). In addition, separate expression of two different ROCK2 shRNAs inhibited phosphorylation of MLC2 at S15 but not Y118 in cytokinesis (Fig. 4h). These results indicate that PKM2-phosphorylated MLC2 at Y118 is required for the interaction between ROCK2 and MLC2 and ROCK2-phosphorylated MLC2 at S15.

MLC2 Phosphorylation by PKM2 is Critical for Cytokinesis. To determine the effect of PKM2-regulated MLC2 on cytokinesis progression, we evaluated live images of late-mitosis U87/ EGFRvIII cells. These cells were infected with the lentivirus that co-expressed GFP and shRNA for PKM2 or MLC2 and the lentivirus that expressed RNAi-resistant WT rPKM2, rPKM2 T45A, WT rMLC2, rMLC2 Y118F or rMLC2 S15A. High viral infection rates, efficient depletion of endogenous PKM2 or MLC2 (Supplementary Fig. 5a), and similar reconstituted expression levels were achieved (Supplementary Fig. 5b). Live cell analyses showed that the mitotic cells expressing WT rPKM2 (Fig. 5a and Supplementary Video 1) and WT rMLC2 (Fig. 5b and Supplementary Video 2) underwent normal and complete cell division. In contrast, a large percentage of cells with reconstituted expression of rPKM2 T45A (Fig. 5a and Supplementary Video 3), rMLC2 Y118F and rMLC2 S15A (Fig. 5b and Supplementary Videos 4 and 5) failed to complete cytokinesis, with regression of cleavage furrow. These results indicate that Aurora B-dependent PKM2 T45 phosphorylation and the subsequent PKM2 phosphorylation-primed MLC2 phosphorylation at S15 by ROCK2 are instrumental to cytokinesis in GBM cells. 
a
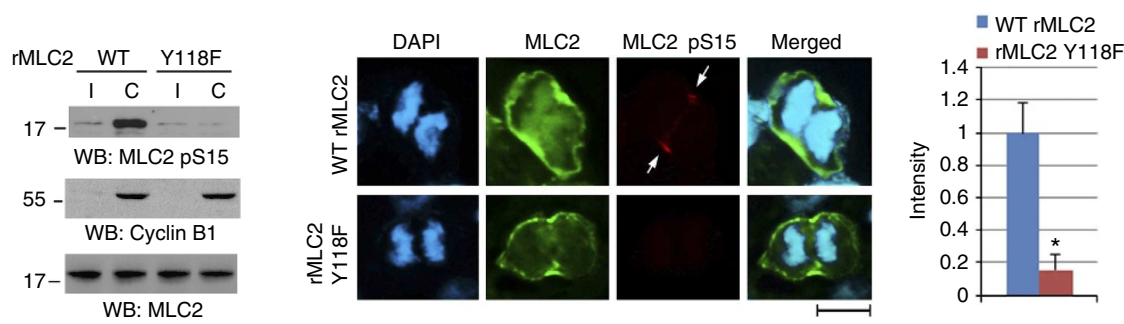

b

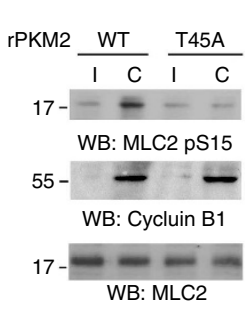

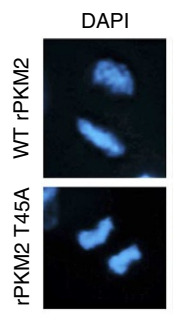

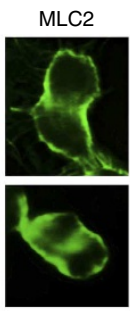

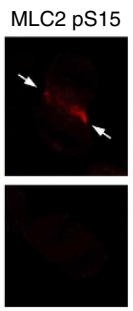

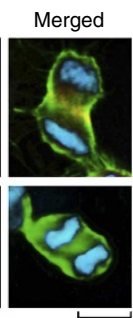

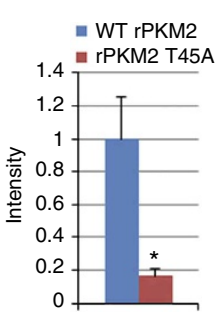

C
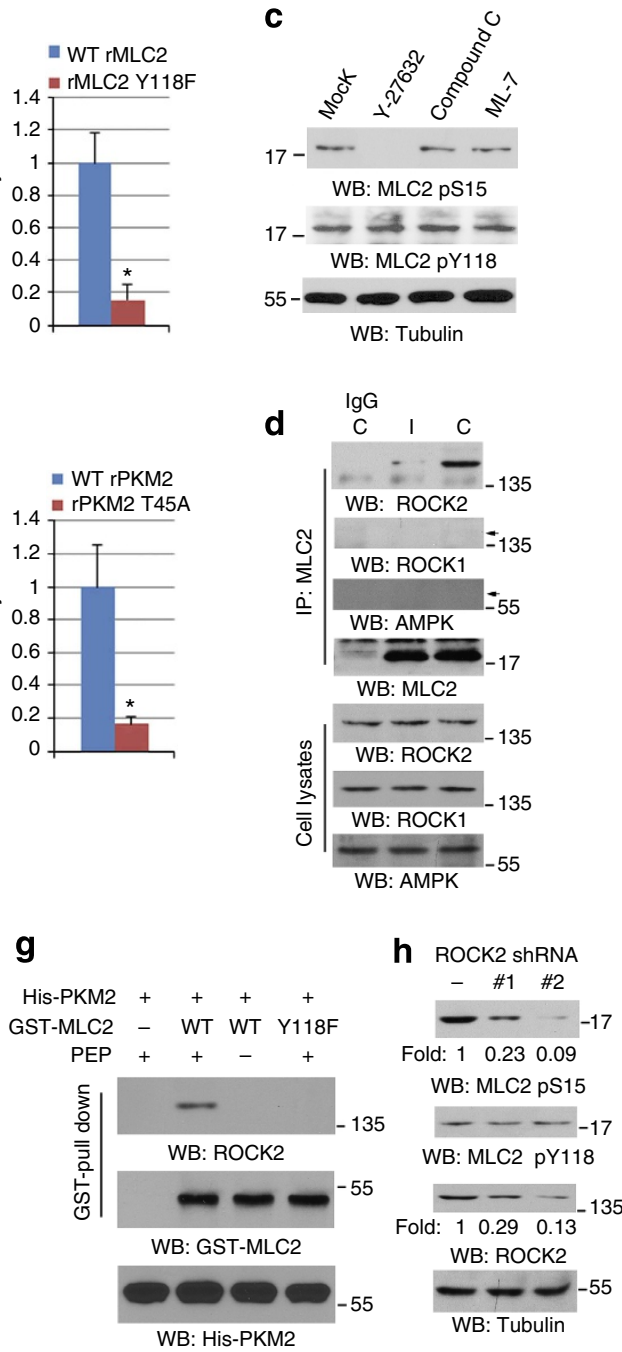

h ROCK2 ShRNA

WB: MLC2 PY118 e

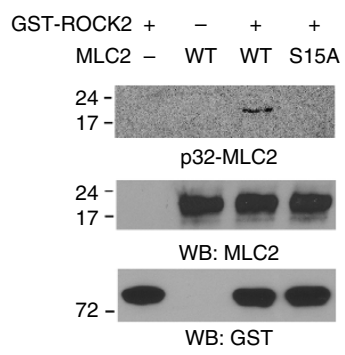

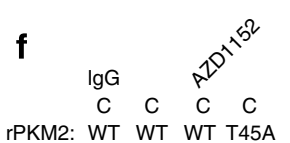

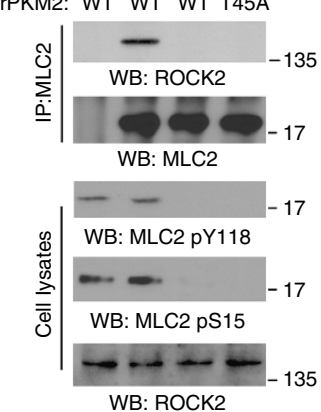

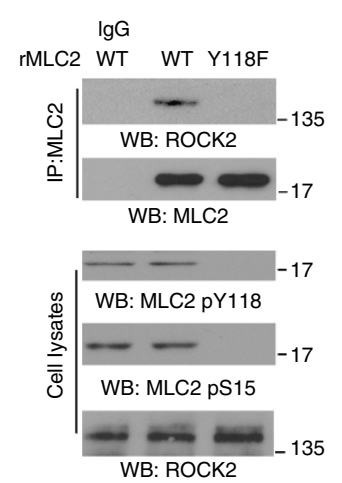

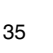

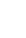

Figure 4 | MLC2 pY118 Primes ROCK2-mediated MLC2 pS15. Immunoblotting analyses were performed with the indicated antibodies. C, cytokinesis; I, interphase (no thymidine release). (a,b) U87 cells with reconstituted expression of WT rMLC2 or rMLC2 Y118F (a), WT rPKM2 or rPKM2 T45A (b) were synchronized by double thymidine block $(2 \mathrm{mM})$ for $43 \mathrm{~h}$, with or without release for $10 \mathrm{~h}$ (left panel). Immunofluorescence analyses of cells in cytokinesis were performed with the indicated antibodies (middle panel). Scale bars, $10 \mu \mathrm{m}$. The relative fluorescence intensity of the indicated proteins in the contractile ring of 100 cells from each cell line was quantified. Data represent the mean \pm s.d. of three independent experiments (right panel). ${ }^{\star} P<0.01$ : statistically significant value in relation to U87 cells with PKM2 depletion and reconstituted expression of WTrPKM2, Student's t-test. (c) U87 cells, synchronized by double thymidine block $(2 \mathrm{mM})$ for $43 \mathrm{~h}$, were released for $9 \mathrm{~h}$, followed by MG132 $(25 \mu \mathrm{M})$ treatment for $1.5 \mathrm{~h}$ to arrest cells at metaphase. MG132 was then replaced with Y-27632 $(10 \mu \mathrm{M})$, compound C $(20 \mu \mathrm{M})$, or ML-7 $(10 \mu \mathrm{M})$ for 30 min before cell harvesting. (d) U87 cells were synchronized by double thymidine block $(2 \mathrm{mM})$ for $43 \mathrm{~h}$, followed by no release or release for $10 \mathrm{~h}$. Immunoprecipitation analyses were performed. (e) In vitro kinase assays were performed by mixing purified recombinant active GST-ROCK2 with purified PKM2-phosphorylated MLC2 for autoradiography. (f) U87 cells with depleted PKM2 or MLC2 and reconstituted expression of WTrPKM2 or rPKM2 T45A (left panel), WT rMLC2 or rMLC2 Y118F (right panel) were synchronized and arrested at metaphase, as described in (c). MG132 was then replaced with AZD1152 (100 nM) for 30 min before cell harvesting (left panel). Immunoprecipitation analyses were performed. (g) WT GST-MLC2 on agarose beads was incubated with purified Aurora B-phosphorylated His-PKM2 protein for a kinase assay in the presence or absence of PEP, which was followed by incubation with the lysate of HeLa cells synchronized by double thymidine block $(2 \mathrm{mM})$ for $43 \mathrm{~h}$ and release for $10 \mathrm{~h}$. (h) U87 cells that expressed a control or two different ROCK2 shRNAs were synchronized by double thymidine block $(2 \mathrm{mM})$ for $43 \mathrm{~h}$ and release for $10 \mathrm{~h}$.

Oncogenic Signalling Promotes PKM2-regulated Cytokinesis. EGFR activation by EGF treatment or EGFRvIII mutant expression in GBM cells significantly upregulated the expression of both PKM2 22 and MLC2 (Fig. 1d). In addition, EGFRvIII expression in U87 cells increased the total phosphorylation levels of PKM2 T45, MLC2 Y118 and MLC2 S15 (Fig. 6a). We performed flow cytometry analyses of synchronized U87 cells with a double thymidine block and release. Figure $6 \mathrm{~b}$ shows that about $35-40 \%$ of U87 cells with depleted endogenous PKM2 and MLC2 and with or without reconstituted expression of rPKM2 T45A, rMLC2 Y118F or rMLC2 S15A had DNA content of more than $4 \mathrm{~N}$, indicating DNA replication in the absence of cell division. In addition, the expression of these PKM2 or MLC2 mutants and depletion of MLC2 inhibited U87 cell proliferation (Fig. 6c). In contrast, reconstituted expression of rPKM2 T45A, rMLC2 Y118F or rMLC2 S15A in U87/EGFRvIII cells resulted in more profound defects in cytokinesis (Fig. 6b) and cell proliferation (Fig. 6c). Furthermore, flow cytometric analyses showed that the expression of rPKM2 T45A, rMLC2 Y118F or rMCL2 S15A resulted in the accumulation of cells in the sub-G1 phase (an indication of apoptotic cells), with decreased number of cells in the G1/S phase (Supplementary Fig. 6a). Notably, overexpression 

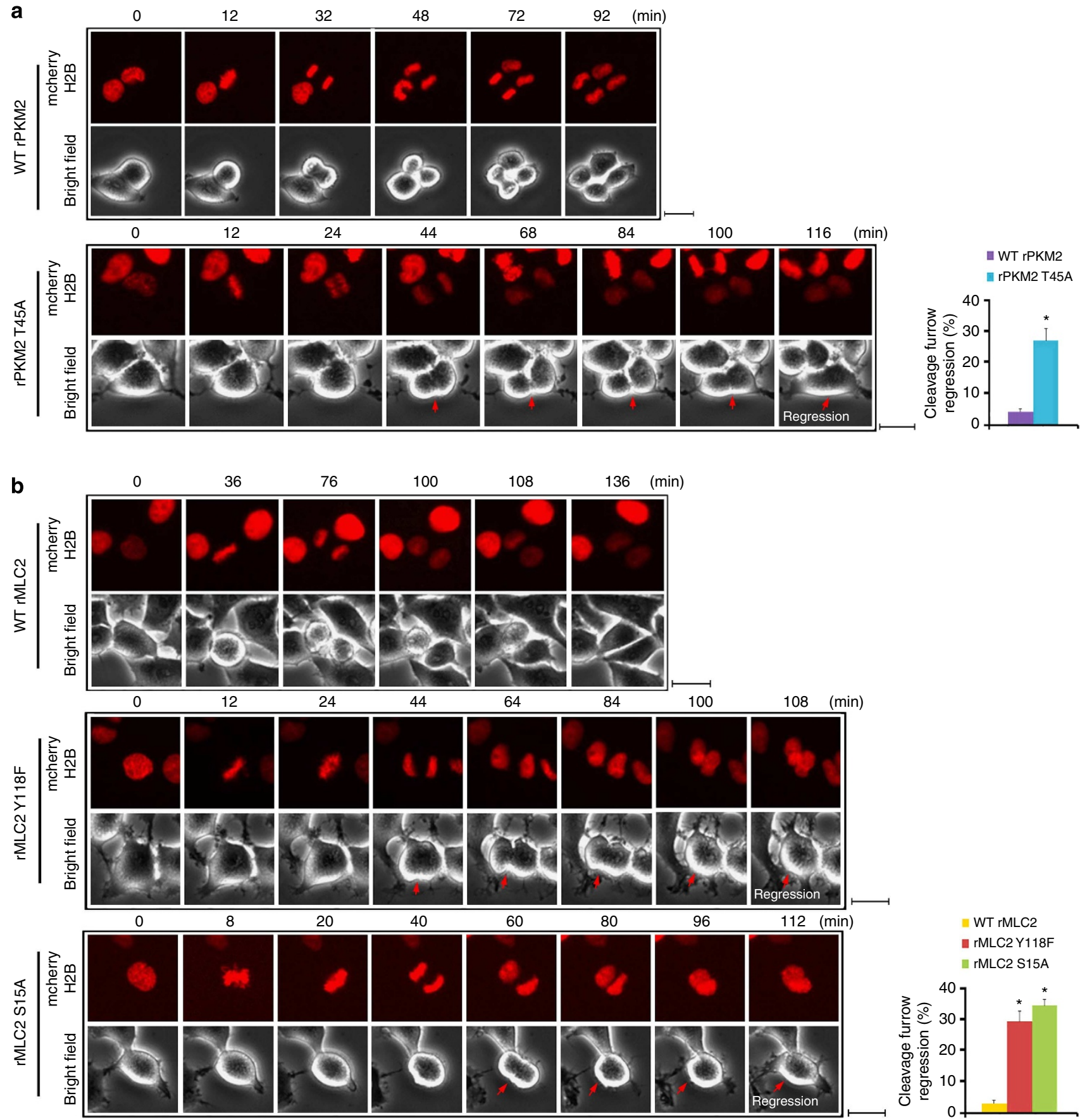

Figure 5 | MLC2 phosphorylation by PKM2 is critical for cytokinesis. (a,b) U87/EGFRvIll cells with PKM2 depletion and reconstituted expression of WT rPKM2 or rPKM2 T45A (a) or with MLC2 depletion and reconstituted expression of WT rMLC2, rMLC2 Y118F or rMLC2 S15A (b) were synchronized by double thymidine block $(2 \mathrm{mM})$ for $43 \mathrm{~h}$ and released for $9 \mathrm{~h}$. Imaging analyses were performed by using a DeltaVision deconvolution microscope with a $\times 20$ lens in a $\mathrm{CO}_{2}$ environment chamber. Pictures were taken at 4-min intervals. Selected time points are shown. Scale bars, $10 \mu \mathrm{m}$. Thirty cells from each cell line were analysed. The data represent the mean \pm s.d. of three independent experiments (right panel). ${ }^{\star} P<0.01$ : statistically significant value in relation to U87/EGFRvIll cells with reconstituted expression of WT rPKM2 (a) or WT rMLC2 (b), Student's $t$-test.

of a phosphorylation-mimic MLC2 S15D mutant largely rescued the cytokinesis defects of rPKM2 T45A-expressed U87 cells, supporting the critical role of MLC2 phosphorylation in PKM2regulated cytokinesis (Supplementary Fig. 6b). In line with the results from U87/EGFRvIII cells, EGFRvIII expression in HeLa cells also led to upregulated expression and phosphorylation levels of PKM2 and MLC2 and exacerbated the cytokinesis defect under the context of PKM2 depletion (Supplementary Fig. 6c). These results indicate that tumour cells with EGFRvIII-enhanced expression of PKM2 and MLC2 increase their dependence on PKM2 cytokinesis regulation.

PKM2 is highly expressed during embryonic development ${ }^{16}$. To determine whether the function of PKM2 in cytokinesis is also shared by rapidly proliferating embryonic cells, we depleted PKM2 or MLC2 from immortalized MEFs and reconstituted their expression with WT rPKM2, rPKM2 T45A, WT rMLC2, rMLC2 Y118F or rMLC2 S15A (Supplementary Fig. 6d). Of note, endogenous expression levels of PKM2 and MLC2 in MEFs 
a

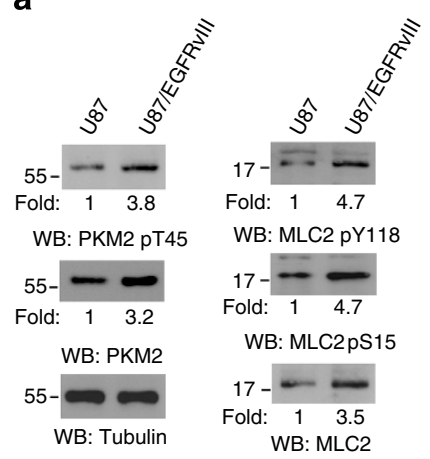

d

\#WT rMLC2 $\quad$ "WT rPKM2 - rMLC2 Y118F $\mid$ rPKM2 T45A $\because \mathrm{rMLC} 2 \mathrm{~S} 15 \mathrm{~A}$

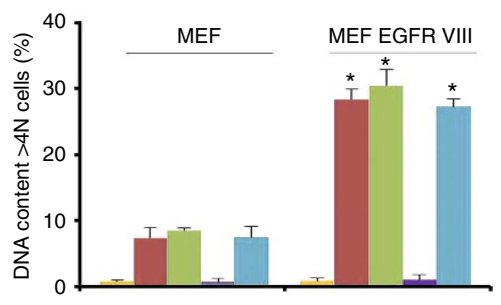

f $\quad W T$ rMLC2 $\quad W T$ rPKM2 - rMLC2 Y118F \|IPKM2 T45A rMLC2 S15A

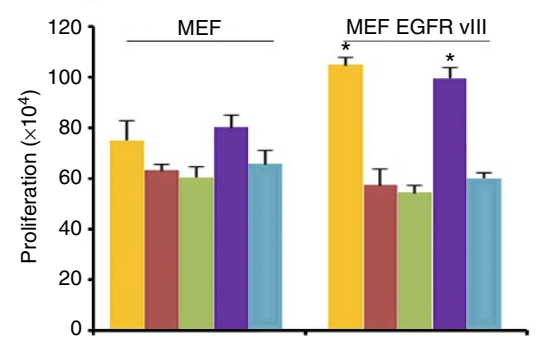

b

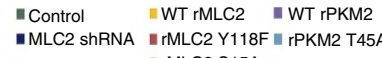
IIMLC2 S15A

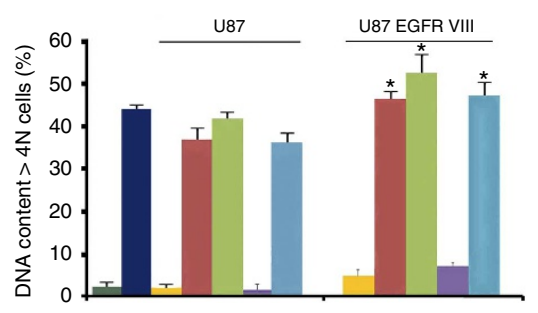

e
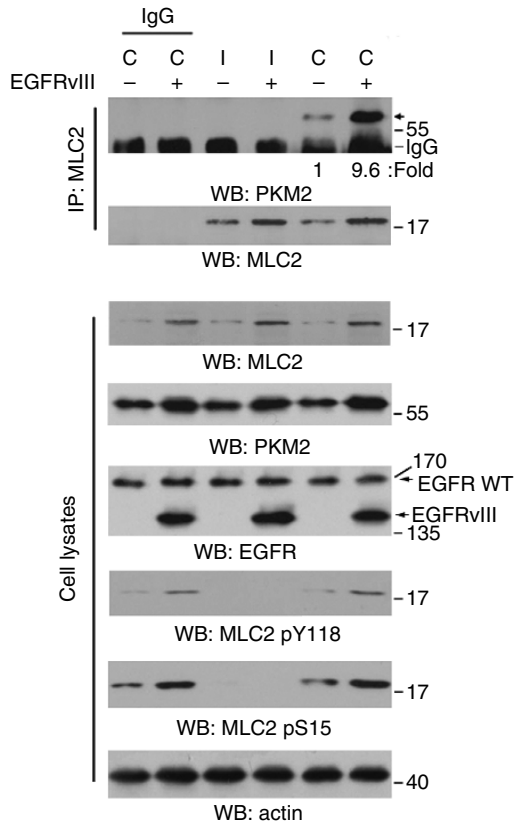

C $\begin{array}{lll}\text { "Control } & \text { WT rMLC2 } & \text { wT rPKM2 } \\ \text { "MLC2 ShRNA } & \text { "rMLC2 Y118F } \| \text { rPKM2 T45A }\end{array}$ IIMLC2 S15A
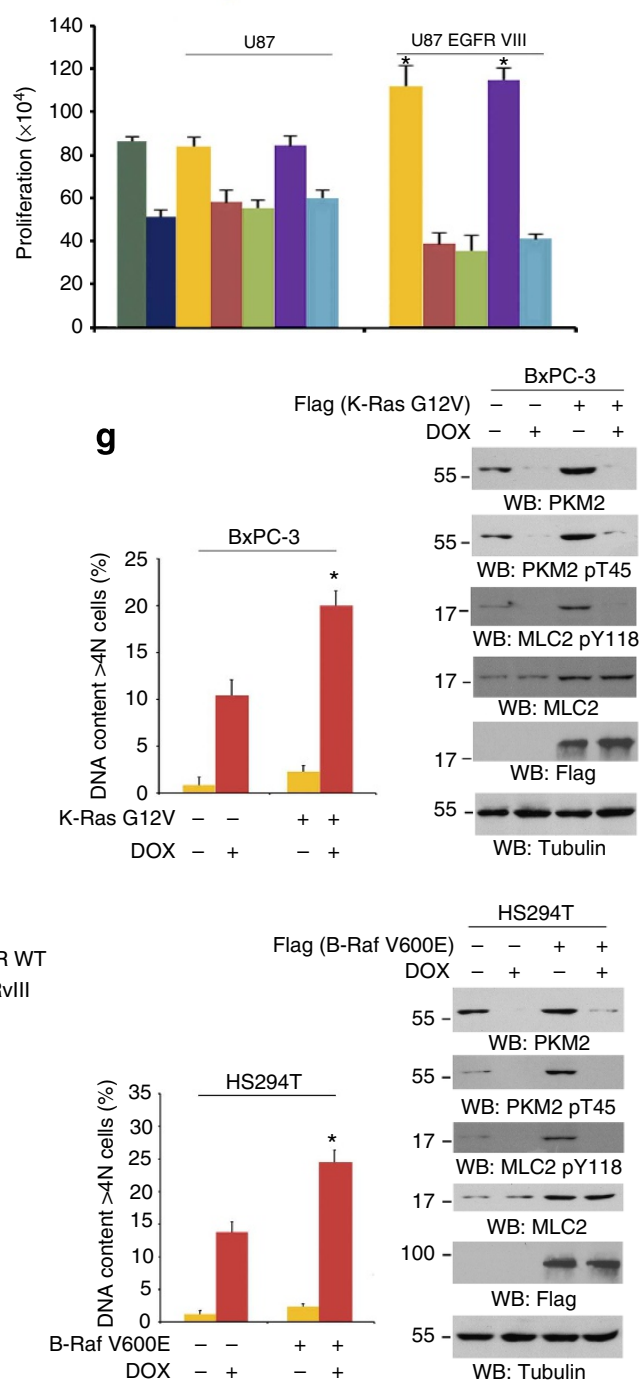

Figure 6 | Oncogenic signalling promotes PKM2-regulated cytokinesis. (a) U87 and U87/EGFRvIll cells, synchronized by thymidine double block $(2 \mathrm{mM})$, were released for $10 \mathrm{~h}$ (anaphase). Immunoblotting analyses were performed with the indicated antibodies. (b,c) U87 and U87/EGFRvIII cells with reconstituted expression of WT rPKM2, rPKM2 T45A, WT rMLC2, rMLC2 Y118F or rMLC2 S15A were synchronized by double thymidine block (2 mM) for $43 \mathrm{~h}$ and released for $36 \mathrm{~h}$. Flow cytometric analyses were performed (b). The cells $\left(2 \times 10^{4}\right)$ were plated and counted 7 days after being seeded in DMEM with $2 \%$ bovine calf serum (c). The data represent the mean \pm s.d. of three independent experiments. ${ }^{\star} P<0.01$ : statistically significant value in relation to parental cells, Student's t-test. (d) MEFs and EGFRvIll-expressing MEFs with reconstituted expression of WT rPKM2, rPKM2 T45A, WT rMLC2, rMLC2 Y118F or rMLC2 S15A were synchronized by double thymidine block $(2 \mathrm{mM})$ for $43 \mathrm{~h}$ and released for $36 \mathrm{~h}$. Flow cytometric analyses were performed. The data represent the mean \pm s.d. of three independent experiments. (e) MEFs and EGFRvIll-expressing MEFs that had been synchronized by thymidine double block $(2 \mathrm{mM}$ ) were unreleased or released for $10 \mathrm{~h}$ (anaphase). Immunoprecipitation and immunoblotting analyses were performed with the indicated antibodies. C, cytokinesis; I, interphase (no thymidine release). (f) The indicated cells $\left(2 \times 10^{4}\right)$ were plated and counted 7 days after being seeded in DMEM with $2 \%$ bovine calf serum. The data represent the mean \pm s.d. of three independent experiments. (g) BxPC3 and K-Ras G12Vexpressing BxPC3 cells (top panel) or HS294T and B-Raf V600E-expressing HS294T cells (bottom panel) were treated with doxycycline (500 ng ml- ${ }^{-1}$ ) for the indicated time periods, as shown in Fig. 1b, to induce PKM2 shRNA expression. Flow cytometric analyses were performed. The data represent the mean \pm s.d. of three independent experiments. Immunoblotting analyses were performed with the indicated antibodies.

were lower than those in U87, U251 and U87/EGFRvIII GBM cells and GSC11 human primary GBM cells (Supplementary Fig. 6e), and the cytokinesis defect rate in MEFs with disrupted PKM2 regulation was significantly lower than that in U87 cells (Fig. 6b,d). Expression of the EGFRvIII mutant in MEFs greatly increased PKM2 and MLC2 expression levels, with an enhanced interaction between PKM2 and MLC2 and phosphorylation levels of MLC2 at Y118 and S15 (Fig. 6e). In addition, the reconstituted expression of rPKM2 T45A, rMLC2 Y118F or rMLC2 S15A in
MEFs/EGFRvIII cells (Supplementary Fig. 6f) heightened the defect of cytokinesis (Fig. 6d) and proliferation (Fig. 6f) in contrast to the expression of these mutants in parental MEFs.

Similar to MEFs, A549 human lung adenocarcinoma cells and HCT116 human colorectal carcinoma cells, which had lower expression levels of MLC2 and PKM2 than did the GBM cells (Supplementary Fig. 6e), had limited defects in cytokinesis and cell proliferation (Supplementary Fig. 6g) after doxycyclineinduced PKM2 depletion (Supplementary Fig. 6h). These results 
suggest that these cells regulate cytokinesis mainly through other isoforms of RMLC protein.

To validate this hypothesis, we determined the role of MRLC1 in cytokinesis in HCT116 cells. We depleted MRLC1 and reconstituted the expression of rMRLC1 T18/S19 phosphorylation-dead mutant (T18/S19A) because T18/S19 phosphorylation of smooth muscle and non-muscle RMLC isoforms is important for actomyosin contraction ${ }^{5-7}$ (Supplementary Fig. 6i, left panel). The expression of rMRLC1 T18/S19A, but not its WT counterpart, led to significant cytokinesis defects (Supplementary Fig. 6i, right panel), indicating that T18/S19 phosphorylation plays a major role in the cytokinesis of HCT116 cells. Reconstituted expression of rMLC2 S15A and rMRLC1 T18/ S19A in HCT116 cells with depleted MLC2 and MRLC1 (Supplementary Fig. 6j, left panel) had an added effect on the cytokinesis defect rate compared with that in HCT116 cells that expressed rMLC2 S15A or rMRLC1 T18/S19A alone (Supplementary Fig. 6j, right panel). These findings indicate that MLC2 S15 and MRLC1 T18/S19 phosphorylations have a non-overlapped function in the regulation of cytokinesis progression. As expected, expression of EGFRvIII increased the phosphorylation levels of both PKM2 and MLC2 in a PKM2dependent manner in A549 and HCT116 cells (Supplementary Fig. 6h). In addition, PKM2 depletion increased the cytokinesis defect rates of EGFRvIII-expressing A549 and HCT116 cells compared with those in their parental cells (Supplementary Fig. 6g). These results indicate that PKM2's limited effect on cytokinesis regulation in tumour cells with low PKM2 and MLC2 expression can be significantly altered after EGFR activation.

To determine whether our findings were limited to EGFR activation, we examined the role of PKM2 in the cytokinesis of tumour cells with K-Ras G12V and B-Raf V600E mutations, which occur in many types of cancer. Figure $6 \mathrm{~g}$ shows that overexpression of $\mathrm{K}$-Ras $\mathrm{G} 12 \mathrm{~V}$ in $\mathrm{BxPC}-3$ human pancreatic cancer cells (with no endogenous Ras mutation) (Fig. 6g, top panel) and B-Raf V600E in HS294T human melanoma cells (with no endogenous Raf mutation) (Fig. 6g, bottom panel) resulted in upregulation of PKM2 and MLC2 expression, PKM2 T45 phosphorylation, and PKM2-dependent MLC2 Y118 phosphorylation and exhibited PKM2 expression-dependent cytokinesis ${ }^{40}$. These results suggest that K-Ras and B-Raf mutations increase the reliance of tumour cells on PKM2-regulated cell division.

MLC2 Phosphorylation by PKM2 Promotes Tumorigenesis. Abnormal cytokinesis affects cell growth and proliferation ${ }^{3}$. Although multi-nucleated cells likely have an aberrant cell metabolism, an in vitro pyruvate kinase assay revealed that PKM2 T45A had similar glycolytic kinase activity to that of its WT counterpart (Supplementary Fig. 7a). In addition, compared with the expression of its WT counterpart, PKM2 T45A expression did not affect the glucose uptake and lactate production in U87/EGFRvIII cells arrested at the G1/S phase (Supplementary Fig. 7b), PKM2-regulated Bub3 Y207 phosphorylation (Supplementary Fig. 7c), chromatid alignment and segregation (Supplementary Fig. 7d), or EGF-induced and PKM2-dependent histone H3 T11 phosphorylation in U87 cells (Supplementary Fig. 7e). These results suggest that PKM2 T45 phosphorylation exerts a specific role in cytokinesis.

To determine the role of PKM2-regulated MLC2 phosphorylation in brain tumour development, we intracranially injected athymic nude mice with PKM2- or MLC2-depleted U87/ EGFRvIII (Supplementary Fig. 5b) and GSC11 (Supplementary Fig. 7f) cells with reconstituted WT rPKM2, rPKM2 T45A, WT rMLC2, rMLC2 Y118F or rMLC2 S15A expression. U87/EGFRvIII (Fig. 7a) and GSC11 (Supplementary Fig. 7g) cells that expressed WT rPKM2 or WT rMLC2 elicited rapid tumorigenesis. In contrast, rPKM2 T45A, rMLC2 Y118F and rMLC2 S15A expression significantly inhibited brain tumour growth (Fig. 7a and Supplementary Fig. 7g) and prolonged mouse survival (Fig. 7b). The expression of Ki-67, a marker of cell proliferation, was reduced in tumour tissue that expressed these mutants (Fig. 7c). Furthermore, rPKM2 T45A expression reduced the phosphorylation levels of MLC2 Y118 and S15, and rMLC2 Y118F expression blocked MLC2 S15 phosphorylation in tumour tissue (Fig. 7c). These results indicate that PKM2-regulated MLC2 phosphorylation and cytokinesis are instrumental in brain tumour development.

\section{Discussion}

PKM2 is known as a classic glycolytic enzyme and is upregulated by growth factor-induced and nuclear factor- $\kappa \mathrm{B}$-regulated $P K M$ gene transcription in tumour cells ${ }^{22}$. PKM2 has critical nuclear functions in directly controlling gene transcription by binding to transcriptional factors, such as $\beta$-catenin and hypoxia-inducible factor $1 \alpha$, and phosphorylating histone $\mathrm{H} 3$ at T11, leading to histone H3 acetylation ${ }^{19,23}$. PKM2-dependent c-Myc and cyclin D1 expression regulates the Warburg effect and G1-S transition, respectively ${ }^{21}$. In addition, PKM2 binds to and phosphorylates Bub3 and regulates correct kinetochore-microtubule attachment, the spindle-assembly checkpoint and accurate chromosome segregation $^{26}$. However, the role of PKM2 in controlling other phases of the cell cycle remains unknown. In this report, we demonstrated that the activation of EGFR, K-Ras and B-Raf resulted in enhanced expression of both PKM2 and MLC2. Importantly, PKM2 T45 is phosphorylated by Aurora B during cytokinesis. This phosphorylation is required for PKM2dependent MLC2 phosphorylation at Y118, leading to the ROCK2-dependent phosphorylation of MLC2 at S15 and cytokinesis progression (Fig. 7d).

Several RMLC isoforms exist in tumour cells, and the phosphorylation of smooth muscle and non-muscle cell isoforms at T18/S19 (which are not conserved in MLC2) and MLC2 at S15 regulates actomyosin contraction by distinct mechanisms ${ }^{5-7}$. The phosphorylation of smooth muscle and non-muscle cell RMLC isoforms results in a conformational change, with enhanced myosin ATPase activity and effects on actin filament formation ${ }^{41}$. In contrast, MLC2 phosphorylation has no significant effect on myosin ATPase activity; instead, it modulates $\mathrm{Ca}^{2}+$ sensitivity and $\mathrm{Ca}^{2+}$ /troponin-dependent force generation, influencing contraction properties ${ }^{6}$. In contrast to the relatively well-studied RMLC S19 phosphorylation regulation, how MLC2 S15 phosphorylation is regulated in tumour cells is largely unknown. We found that MLC2 expression was significantly increased in tumour cells that expressed active EGFRvIII, K-Ras G12V and B-Raf V600E mutants. In addition, the sequential interaction between PKM2 and MLC2 and the interaction between MLC2 and ROCK2 are dependent on the primed phosphorylation of PKM2 and MLC2, respectively. These results strongly suggest that phosphorylation-mediated structural changes of proteins or the creation of protein-binding motifs promotes subsequent protein interactions. PKM2 does not associate with ROCK2, suggesting that it disassociates from the ROCK2 and MLC2 complex after phosphorylating MLC2 Y118. These findings highlight the role of PKM2-involved protein posttranslational modifications in precisely controlling cytokinesis. The added effect of MLC2 S15A and MRLC1 T18/S19A expression on blocking cytokinesis progression indicates that a coordinated regulation of different RMLC isoforms is required for the fully executed constriction of the contractile ring and cytokinesis completion. 
a

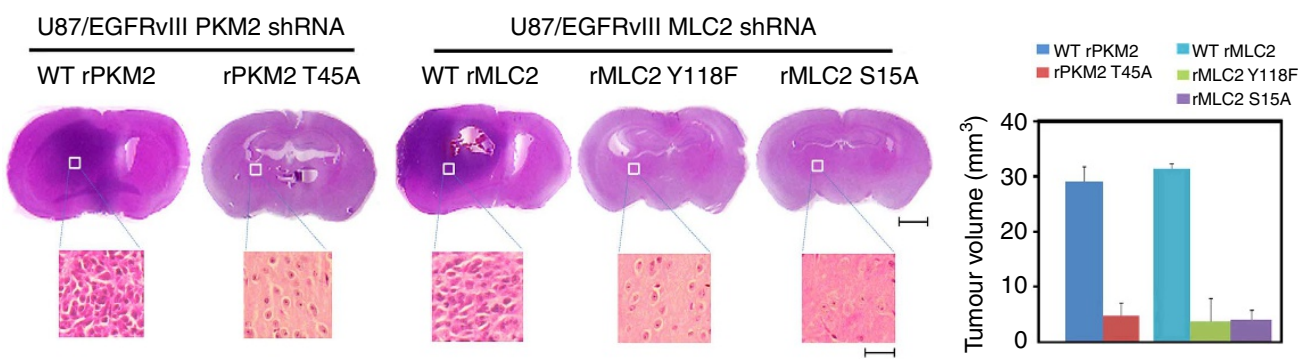

b

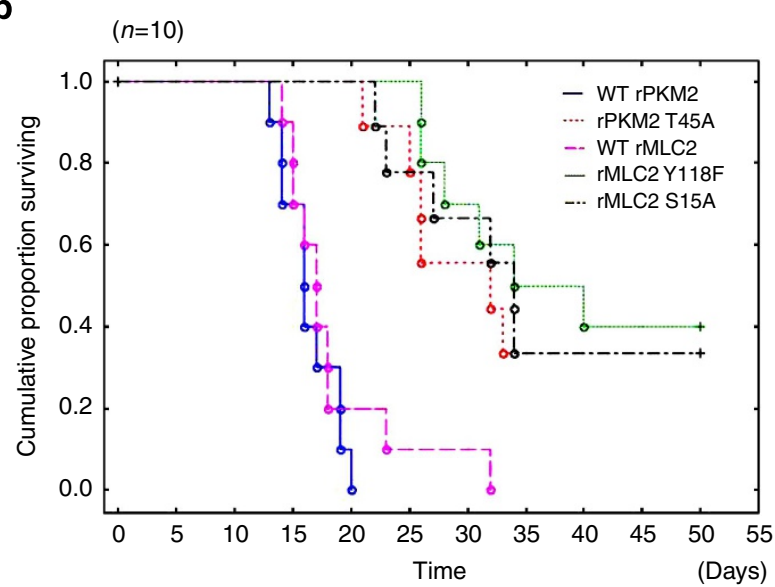

C

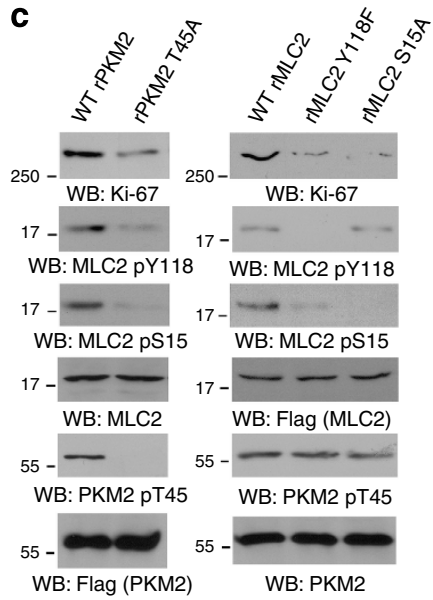

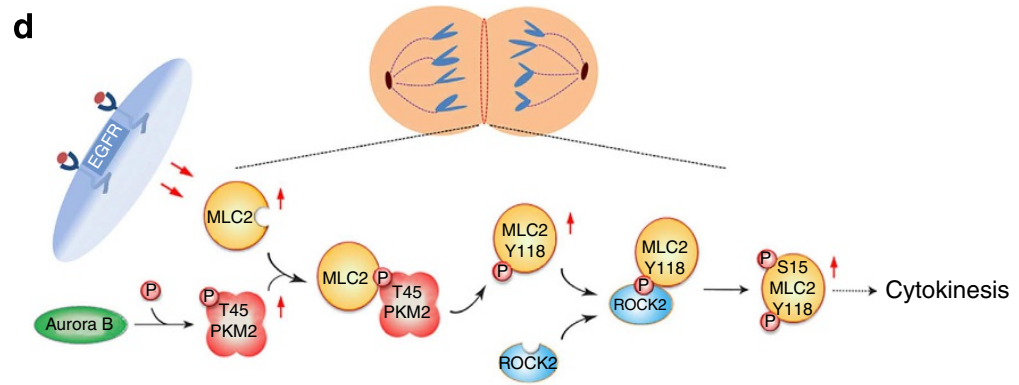

Figure 7 | MLC2 phosphorylation by PKM2 promotes tumorigenesis. (a,c) U87/EGFRvIII $\left(5 \times 10^{5}\right)$ cells with PKM2 depletion and reconstituted expression of WT rPKM2 or rPKM2 T45A or with MLC2 depletion and reconstituted expression of WT rMLC2, rMLC2 Y118F or rMLC2 S15A were intracranially injected into athymic nude mice. The mice were killed 14 days after injection of cells and examined for tumour growth. Haematoxylin-andeosin-stained coronal brain sections show representative tumour xenografts. Scale bars for original images, $0.2 \mathrm{~cm}$; Scale bars for amplified images, $50 \mu \mathrm{m}$. Tumour volumes were measured using length (a) and width (b) and calculated using the equation: $V=a b^{2} / 2$. The data represent the means $\pm \mathrm{s}$.d. of seven mice (right panel). The tumour tissues normalized with expression levels of Flag-tagged rPKM2 or rMLC2 were immunoblotted with the indicated antibodies (c). (b) U87/EGFRvIII $\left(5 \times 10^{5}\right)$ cells with PKM2 depletion and reconstituted expression of WT rPKM2 or rPKM2 T45A or with MLC2 depletion and reconstituted expression of WT rMLC2, rMLC2 Y118F or rMLC2 S15A were intracranially injected into athymic nude mice. Data were presented by the Kaplan-Meier plots of 10 mice. (d) A mechanism of PKM2-regulated cytokinesis. Aurora B-phosphorylated PKM2 at T45 results in PKM2 localization and its interaction with MLC2 at the equator/contractile ring region of mitotic cells, where PKM2 phosphorylates MLC2 at Y118. MLC2 Y118 phosphorylation primes ROCK2 binding to MLC2, ROCK2-dependent MLC2 S15 phosphorylation and subsequent cytokinesis progression.

Aurora kinase B localizes at both the contractile ring/cleavage furrow region and the central spindle area during cytokinesis ${ }^{42}$. Intriguingly, PKM2 partially localizes at the contractile ring region as well as the central spindle area, suggesting that PKM2 is involved in both cytokinesis and spindle morphologic alteration.

Our results suggest that PKM2-regulated cytokinesis occurs in certain types of tumours. It is known that cell cycle progression and cell proliferation can be promoted by distinct signal transduction that is induced by oncogene activation, tumour suppressor gene deficiency, or both. Published evidence demonstrates that PKM2 regulation is signalling dependent, and the roles of PKM2 in tumorigenesis vary on the basis of the signalling context. For instance, recent reports showed that PKM2 deficiency in mice did not block Brcal loss-driven mammary tumour formation but inhibited cyclin D1 expression and leukemia initiation induced by BCR-ABL and MLL-AF9 (refs 43,44). These findings suggest that PKM2, which is not highly expressed in every type of human cancer, regulates tumour development in a signalling context-dependent manner. In line with these observations, we showed that expression of EGFRvIII in GBM cells, K-Ras G12V in pancreatic cancer cells and B-Raf V600E in melanoma cells resulted in upregulation of PKM2 and MLC2 expression, Aurora B-mediated PKM2 T45 phosphorylation, PKM2-dependent MLC2 Y118 phosphorylation and MLC2 Y118-dependent cytokinesis. These results suggest that tumour cells with activated EGFR, K-Ras or B-Raf compared with 
other cells without activation of these oncogenes develop a new pattern of molecular signatures for regulating cell proliferation: enhanced PKM2 expression to regulate glycolysis and prepare building blocks for cell proliferation and enhanced PKM2 and MLC2 expression to govern the progression of cytokinesis. These changes in the molecular signature enable these tumour cells to coordinate their metabolism and cycle progression through PKM2 (refs 21,23,45,46).

Human PKM2 is expressed in early fetal tissues and decreases immediately after birth. In many cancer cells, it is overexpressed $^{47-49}$. In this study, we found that it had a limited effect on cytokinesis in MEFs and tumour cells (such as HCT116 cells) with low levels of PKM2 expression, which is in line with the results of a report that PKM2 depletion in HCT116 cells has limited effect on tumour growth ${ }^{50}$. Of note, cytokinesis of HCT116 cells is largely dependent on MRLC1 regulation. However, activation of EGFR increases the cell dependence on PKM2-regulated cytokinesis by increasing the expression levels of PKM2 and MLC2 and PKM2-mediated MLC2 phosphorylation. These results suggest that PKM2 participates in cytokinesis of certain types of normal and tumour cells in a manner dependent on the level and duration of activation of growth factor receptors, such as EGFR. Importantly, tumour cells with constitutively activated oncogenic signalling induced by EGFR, K-ras and B-Raf significantly upregulate PKM2 and enhances the activity of upregulated MLC2. The disruption of PKM2-dependent MLC2 phosphorylation in GBM cells leads to polyploidy and inhibits tumour cell proliferation and tumour development.

Thus, in our study, we identified a novel regulatory mechanism for cell cycle progression. Our findings highlight the nonmetabolic function of PKM2 as a protein kinase that controls cell division and may provide a molecular basis for improving the diagnosis and treatment of tumours with upregulated PKM2.

\section{Methods}

Cell culture and synchronization. U87, U251 and MEFs were from ATCC and were maintained in Dulbecco's modified Eagle's medium (DMEM) supplemented with $10 \%$ bovine calf serum (HyClone, Logan, UT). Human primary GSC11 GBM cells were maintained in DMEM/F-12 50/50 supplemented with B27, EGF $\left(10 \mathrm{ng} \mathrm{ml}^{-1}\right)$ and basic fibroblast growth factor $\left(10 \mathrm{ng} \mathrm{ml}^{-1}\right)$.

Confluent cells (30-40\%) were washed twice with phosphate-buffered saline (PBS), treated with $2 \mathrm{mM}$ thymidine for $17 \mathrm{~h}$, washed twice in PBS again, released in complete medium containing $10 \mu \mathrm{M}$ deoxycytidine for $9 \mathrm{~h}$, treated with $2 \mathrm{mM}$ thymidine for $17 \mathrm{~h}$, released in complete medium with $10 \mu \mathrm{M}$ deoxycytidine and assayed.

After the double thymidine block, cells were washed twice with PBS, released in complete medium for $9 \mathrm{~h}$ and treated with MG132 $(25 \mu \mathrm{M})$ for $1.5 \mathrm{~h}$. Cells were arrested in metaphase.

Materials. Rabbit polyclonal antibodies that recognize MLC2 (1:500 for immunoblotting (IB) analyses ), phospho-MLC2 S15 (1:400 for IB), phospho-MLC2 Y118 (1:400 for IB), PKM1 (1:1,000 for IB), PKM2 (1:1,000 for IB) and cyclin B1 (1:1,000 for IB) were obtained from Signalway Antibody (College Park, MD). A mouse antibody that recognizes MLC2 (1:500 for IB, 1:100 for IP) was obtained from Santa Cruz (San Jose, CA). Antibodies that recognize Aurora B (1:500 for IB), ROCK2 (1:1,000 for IB), MLC kinase (1:1,000 for IB) and AMPK (1:1,000 for IB) were purchased from Abcam (Boston, MA). Rabbit polyclonal antibodies against MRLC1 (MYL9) (1:1,000 for IB), phospho-MRLC1 (MYL9) S19 (1:500 for IB), ACC (1:1,000 for IB) and phospho-ACC S79 (1:500 for IB) were from Cell Signaling Technology (Beverly, MA). GST-Purified ROCK2 protein, purified GST-Aurora B protein, thymidine and mouse monoclonal antibodies for tubulin, Flag (1:5,000 for IB, 1:500 for IP), GST (1:1,000 for IB) and His (1:1,000 for IB) were purchased from Sigma (St Louis, MO). Hygromycin, puromycin, DNase-free RNase A and propidium iodide were purchased from EMD Biosciences (San Diego, CA). DAPI (1:3,000 for IB); Alexa Fluor 488 (1:400 for IF), 594 (1:400 for IF) and 633 goat anti-rabbit (1:400 for IF) antibodies; and Alexa Fluor 488 (1:400 for IF), 594 (1:400 for IF) and 633 goat anti-mouse (1:400 for IF) antibodies were from Molecular Probes (Eugene, OR). HyFect transfection reagent was from Denville Scientific (Metuchen, NJ). GelCode blue stain reagent was obtained from Pierce (Rockford, IL). GST-ROCK2 and GST-Aurora B were purchased from Sigma (St Louis, MO).
Transfection. Cells were plated at a density of $4 \times 10^{5} / 60-\mathrm{mm}$ dish $18 \mathrm{~h}$ before transfection. The transfection was performed using HyFect reagent (Denville Scientific) according to the vendor's instructions.

Mass spectrometry analysis. Aurora B-phosphorylated PKM2 and PKM2phosphorylated MLC2 were acetone-precipitated in vitro at $-20^{\circ} \mathrm{C}$ overnight and re-suspended in $50 \mathrm{mM}$ ammonium bicarbonate buffer containing Rapigest (Waters Corp, MA). The sample was heated to $95^{\circ} \mathrm{C}$ for $10 \mathrm{~min}$ and allowed to cool before $100 \mathrm{ng}$ of sequencing-grade modified trypsin (Promega, Madison, WI) was added. The digestion proceeded overnight at $37^{\circ} \mathrm{C}$ and was analysed by LC-MS/MS using an Obitrap-Elite mass spectrometer (Thermo Fisher Scientific, Waltham, MA).

Proteins were identified by comparing the fragment spectra against those in the SwissProt protein database (EBI) using Mascot v.2.3 (Matrix Science, London, UK) and Sequest v.1.20 via Proteome Discoverer v.1.3 (Thermo Fisher Scientific) software. Phosphopeptide matches were analysed using PhosphoRS via Proteome Discoverer and manually curated ${ }^{51}$.

Immunoprecipitation and immunoblotting analysis. Proteins were extracted from cultured cells using a modified buffer, consisting of $50 \mathrm{mM}$ Tris- $\mathrm{HCl}$ (pH 7.5), $1 \%$ Triton $\mathrm{X}-100,150 \mathrm{mM} \mathrm{NaCl}, 1 \mathrm{mM}$ dithiothreitol, $0.5 \mathrm{mM}$ EDTA, $0.1 \mathrm{mM}$ phenylmethylsulfonyl fluoride, leupeptin $\left(12 \mathrm{mg} \mathrm{ml}^{-1}\right)$, aprotinin $\left(20 \mu \mathrm{g} \mathrm{ml}^{-1}\right)$, $100 \mu \mathrm{M}$ sodium vanadate, $100 \mu \mathrm{M}$ sodium pyrophosphate and $1 \mathrm{mM}$ sodium fluoride. Cell extracts were clarified by centrifugation at 12,000 r.p.m., and the supernatants were subjected to immunoprecipitation and immunoblotting with the corresponding antibodies ${ }^{52}$. Representative original images of immunoblotting results for Figs 1-3 are shown in Supplementary Figs 8-10.

Cell proliferation assay. Cells $\left(2 \times 10^{4}\right)$ were plated and counted 7 days after being seeded in DMEM with $2 \%$ bovine calf serum. The data represent the mean \pm standard deviation (s.d.) of three independent experiments.

DNA constructs and mutagenesis. pcDNA 3.1/hygro (+ ) MLC2 Y118F, Y152F, S15A and PKM2 T45A were created using the QuikChange site-directed mutagenesis kit (Stratagene, Santa Clara, CA). The pGIPZ controls were generated with control oligonucleotide $5^{\prime}$-GCTTCTAACACCGGAGGTCTT- $3^{\prime}$ or $5^{\prime}$-GCCC GAAAGGGTTCCAGCTTA-3'. pGIPZ human PKM2 shRNA was generated with $5^{\prime}$-CATCTACCACTTGCAATTA-3' ${ }^{\prime}$ oligonucleotide that targeted exon 10 of the PKM2 transcript. pGIPZ human MLC2 shRNA was generated with oligonucleotide $5^{\prime}$-ACATTGTGAGGAACACAGT- ${ }^{\prime}$. pGIPZ human MYL9 shRNA was generated with oligonucleotide $5^{\prime}$-TGAGGATGCGGGTGAACTC-3'. Two pGIPZ human ROCK2 shRNAs were generated with oligonucleotides $5^{\prime}$-AGTAATAACAGAT TCTTTG- $3^{\prime}$ and $5^{\prime}$-AACTTGGCAGTCTCCAGCA- ${ }^{\prime}$. pGIPZ mouse PKM2 shRNA was generated with $5^{\prime}$-TCCTCGAATAGCTGCAAGT- $3^{\prime}$ oligonucleotide that targeted exon 10 of the PKM2 transcript. pGIPZ mouse MLC2 shRNA was generated with oligonucleotide $5^{\prime}$-TGTGTGGTCAGCATCTCCC- $3^{\prime}$. PCRamplified bacteria (Escherichia coli) PEPCK DNA was cloned into pCold vector between KpnI and EcoRI.

In vitro kinase assays. The kinase reactions were performed as described previously ${ }^{53}$. In brief, bacterially purified recombinant PKM2 $(200 \mathrm{ng})$ was incubated with MLC2 $(100 \mathrm{ng})$ in kinase buffer $(50 \mathrm{mM}$ Tris- $\mathrm{HCl}(\mathrm{pH} 7.5), 100 \mathrm{mM} \mathrm{KCl}$, $50 \mathrm{mM} \mathrm{MgCl}_{2}, 1 \mathrm{mM} \mathrm{Na}_{3} \mathrm{VO}_{4}, 1 \mathrm{mM}$ dithiothreitol, $5 \%$ glycerol, $0.5 \mathrm{mM}^{32} \mathrm{P}-\mathrm{PEP}$, and $0.05 \mathrm{mM}$ fructose-1.6-bisphosphate in $25 \mu \mathrm{l}$ at $25^{\circ} \mathrm{C}$ for $1 \mathrm{~h}$. The reactions were terminated by adding SDS-PAGE (SDS-polyacrylamide gel electrophoresis) loading buffer and heating to $100^{\circ} \mathrm{C}$

Aurora B and ROCK2 kinase activity assays were performed using the HTScan Aurora B and ROCK2 kinase assay kit, following the manufacturer's instructions (Cell Signaling Technology, Beverly, MA).

32P-PEP preparation. The assays were performed with purified His-PEPCK in $0.05-\mathrm{ml}$ volumes, which contained $160 \mu \mathrm{Ci}{ }^{32} \mathrm{P}$-ATP, $20 \mathrm{mM}$ oxaloacetate, $5 \mathrm{mM}$ $\mathrm{MgCl}_{2}, 50 \mathrm{mM}$ potassium phosphate buffer ( $\mathrm{pH} 7.5$ ), $12.5 \mathrm{mM} \mathrm{KF}$ and $2 \mathrm{mM}$ dithioerythritol. Assays were carried out at $37^{\circ} \mathrm{C}$ for $0.5 \mathrm{~h} .{ }^{32} \mathrm{P}-\mathrm{PEP}$ was separated from ${ }^{32} \mathrm{P}$-ATP using anion exchange chromatography. Bound product was washed with reaction buffer, and the ${ }^{32} \mathrm{P}$-labelled PEP eluted stepwise using $0.3 \mathrm{mM}$ triethylammonium bicarbonate (TEAB) $(\mathrm{pH}$ 8.5). Determining ATP and PEP concentrations in each fraction confirmed the separation of PEP from ATP ${ }^{54,55}$.

The activity of bacterially purified WT PKM2 $(0.1 \mu \mathrm{g})$ and PKM2 T45A $(0.1 \mu \mathrm{g})$ towards PEP was measured using a pyruvate kinase assay (BioVision, Mountain View, CA), according to the manufacturer's instructions. The data represent the mean \pm s.d. of three independent experiments.

Quantitative RT-PCR. A quantitative real-time PCR analysis was performed as described previously ${ }^{23}$ and was used to measure relative mRNA levels; the value was calculated according to the relative amount of internal control. The following primer pairs were used: human MYL2, 5' -ACCATTCTCAACGC 
ATTCAA-3' (forward) and 5'-CATCTGGTCAACCTCCTCCT-3' (reverse); human MYL9, $5^{\prime}$-CTTCACCATGTTCCTCACCA- $3^{\prime}$ (forward) and $5^{\prime}$-GGTCCTC ATGGATGAAACCT-3' (reverse); human MYLB12A/B, 5'-TGCAACATCCAATG TGTTTG- $3^{\prime}$ (forward) and $5^{\prime}$-CATGCAAATCTTCCTTGTCG- $3^{\prime}$ (reverse); mouse MYL2, 5'-GTGTTCCTCACGATGTTTGG-3' (forward) and 5' -AGTCAGCCTTC AGTGACCCT-3' (reverse); mouse MYL9, 5'-GAGGGCTACGTCCAATGTCT- $3^{\prime}$ (forward) and $5^{\prime}$-GTCGTGCAGGTCCTCCTTAT-3' (reverse); and mouse MYLB12A/B, 5'-CACCATCCAGGAGGATTACC-3' (forward) and 5'-CTTCAGG ATGCGTGTGAACT-3' (reverse).

Flow cytometry analysis. Cells $\left(1 \times 10^{6}\right)$ were fixed in $70 \%$ ethanol on ice for $3 \mathrm{~h}$, spun down and incubated for $1 \mathrm{~h}$ at $37^{\circ} \mathrm{C}$ in PBS with DNase-free RNase A $\left(100 \mu \mathrm{g} \mathrm{ml}^{-1}\right)$ and propidium iodide $\left(50 \mu \mathrm{g} \mathrm{ml}^{-1}\right)$. Cells were then analysed by fluorescence-activated cell sorting.

Recombinant protein purification. Wild-type and mutant GST-PKM2, HisPKM2, His-PKM1, His-MLC2, GST-MLC2 and His-PEPCK were expressed in bacteria. Bacteria cultures were grown at $37^{\circ} \mathrm{C}$ to an $\mathrm{OD}_{600}$ of $\sim 0.6$ before inducing with $0.4 \mathrm{mM}$ IPTG for $3 \mathrm{~h}$. Cell pellets were collected, resuspended in $9 \mathrm{ml}$ lysis buffer (50 mM Tris-Cl pH 8.0, $120 \mathrm{mM} \mathrm{NaCl}, 1 \mathrm{mM}$ DTT, $1 \mathrm{mM}$ PMSF, $1 \mathrm{mM}$ benzamidine, $20 \mu \mathrm{g} \mathrm{ml}^{-1}$ leupeptin and $1.0 \mu \mathrm{g} \mathrm{ml}^{-1}$ aprotinin) and lysed by sonication. Triton X-100 was added to the lysates at a final concentration of $1 \%$ and left on ice for $20 \mathrm{~min}$, before centrifugation at 10,000 r.p.m. for $10 \mathrm{~min}\left(4^{\circ} \mathrm{C}\right)$ Cleared lysates were then bound to glutathione-agarose or Ni-NTA agarose for $1.0 \mathrm{~h}$, with rolling at $4^{\circ} \mathrm{C}$. Beads were washed extensively with lysis buffer before eluting for $20 \mathrm{~min}$ in the lysis buffer ( $\mathrm{pH} 7.5$ ) with $20 \mathrm{mM}$ glutathione for GST fusion proteins or in an elution buffer $(0.5 \mathrm{mM}$ imidazole, $0.5 \mathrm{M} \mathrm{NaCl}, 20 \mathrm{mM}$ Tris$\mathrm{HCl} \mathrm{pH} \mathrm{7.9)} \mathrm{for} \mathrm{His-tagged} \mathrm{proteins.} \mathrm{Eluted} \mathrm{proteins} \mathrm{were} \mathrm{then} \mathrm{dialysed} \mathrm{extensively}$ against $20 \mathrm{mM}$ Tris- $\mathrm{Cl} \mathrm{pH} \mathrm{8.0,50} \mathrm{mM} \mathrm{NaCl,} 10 \%$ glycerol and $1 \mathrm{mM} \mathrm{DTT}^{56}$.

IF analysis. Cells were fixed and incubated with primary antibodies: Flag $(1: 3,000)$, PKM2 (1:400), MLC2 (1:400), MLC2 pY118 (1:200), MLC2 pS15 (1:100), Alexa Fluor dye-conjugated secondary antibodies (1:400) and DAPI (1:3,000), according to standard protocols. Cells were evaluated using a deconvolution microscope (Zeiss, Thornwood, NY) with a 63-Å oil immersion objective. Axio Vision software from Zeiss was used to de-convolute Z-series images. For live-cell time-lapse microscopy, we cultured cells in 35-mm glass-bottomed dishes. Cells were imaged using a DeltaVision deconvolution microscope or a Spin Disk confocal microscope with a $\times 20$ lens in a $\mathrm{CO}_{2}$ environment chamber. Pictures were taken at 4-min intervals.

Immunohistochemical analysis. Mouse tumour tissues were fixed and stained with Mayer's haematoxylin and eosin (H\&E) (Biogenex Laboratories, Fremont, CA). The slides were mounted using Fluorogel with Tris buffer (Electron Microscopy Sciences, Hatfield, PA).

Intracranial injection. We intracranially injected 4-week-old athymic nude mice with (1) $5 \times 10^{5}$ U87/EGFRvIII or GSC11 cells that expressed PKM2 shRNA with WT rPKM2 or rPKM2 T45A or (2) U87/EGFRvIII or GSC11 cells that expressed MLC2 shRNA with WT rMLC2, rMLC2 Y118F or rMLC2 S15A (in $5 \mu$ of DMEM per mouse). The injections were performed as described in a previous publication ${ }^{19}$. We divided the mice into two groups. One group (seven mice for each subgroup) was used for immunoblotting or histochemical analyses, and the mice were killed 2 weeks after glioma cell injection. The brain of each mouse was harvested, frozen by liquid nitrogen for immunoblotting analyses or fixed in $4 \%$ formaldehyde for paraffin embedding. Protein expression levels in tumour tissues isolated from the centre of the tumours were normalized by immunoblotting analyses with an anti-FLAG antibody. Tumour formation and phenotype were determined by a histological analysis of H\&E-stained sections.

The other group (10 mice for each subgroup) was monitored for survival. The duration of mouse survival was analysed by STATISTICA software and was represented by Kaplan-Meier plots.

The use of animal was approved by the institutional review board at MD Anderson Cancer Center.

\section{References}

1. Pollard, T. D. Mechanics of cytokinesis in eukaryotes. Curr. Opin. Cell. Biol. 22, 50-56 (2010).

2. Green, R. A., Paluch, E. \& Oegema, K. Cytokinesis in animal cells. Annu. Rev. Cell Dev. Biol. 28, 29-58 (2012).

3. Sagona, A. P. \& Stenmark, H. Cytokinesis and cancer. FEBS Lett. 584, 2652-2661 (2010).

4. Glotzer, M. The molecular requirements for cytokinesis. Science 307, 1735-1739 (2005).

5. Matsumura, F., Totsukawa, G., Yamakita, Y. \& Yamashiro, S. Role of myosin light chain phosphorylation in the regulation of cytokinesis. Cell Struct. Funct. 26, 639-644 (2001)
6. Scruggs, S. B. \& Solaro, R. J. The significance of regulatory light chain phosphorylation in cardiac physiology. Arch. Biochem. Biophys. 510, 129-134 (2011).

7. Iwasaki, T., Murata-Hori, M., Ishitobi, S. \& Hosoya, H. Diphosphorylated MRLC is required for organization of stress fibers in interphase cells and the contractile ring in dividing cells. Cell Struct. Funct. 26, 677-683 (2001).

8. Matsumura, F. Regulation of myosin II during cytokinesis in higher eukaryotes. Trends Cell Biol. 15, 371-377 (2005)

9. Altenberg, B. \& Greulich, K. O. Genes of glycolysis are ubiquitously overexpressed in 24 cancer classes. Genomics 84, 1014-1020 (2004).

10. Majumder, P. K. et al. mTOR inhibition reverses Akt-dependent prostate intraepithelial neoplasia through regulation of apoptotic and HIF-1-dependent pathways. Nat. Med. 10, 594-601 (2004).

11. Noguchi, T., Inoue, H. \& Tanaka, T. The M1- and M2-type isozymes of rat pyruvate kinase are produced from the same gene by alternative RNA splicing. J. Biol. Chem. 261, 13807-13812 (1986).

12. David, C. J., Chen, M., Assanah, M., Canoll, P. \& Manley, J. L. HnRNP proteins controlled by c-Myc deregulate pyruvate kinase mRNA splicing in cancer. Nature 463, 364-368 (2010).

13. Christofk, H. R. et al. The M2 splice isoform of pyruvate kinase is important for cancer metabolism and tumour growth. Nature 452, 230-233 (2008).

14. Koppenol, W. H., Bounds, P. L. \& Dang, C. V. Otto Warburg's contributions to current concepts of cancer metabolism. Nat. Rev. Cancer 11, 325-337 (2011).

15. Cairns, R. A., Harris, I. S. \& Mak, T. W. Regulation of cancer cell metabolism. Nat. Rev. Cancer 11, 85-95 (2011).

16. Vander Heiden, M. G., Cantley, L. C. \& Thompson, C. B. Understanding the Warburg effect: the metabolic requirements of cell proliferation. Science 324, 1029-1033 (2009).

17. Hsu, P. P. \& Sabatini, D. M. Cancer cell metabolism: Warburg and beyond. Cell 134, 703-707 (2008).

18. Lv, L. et al. Acetylation targets the M2 isoform of pyruvate kinase for degradation through chaperone-mediated autophagy and promotes tumor growth. Mol. Cell 42, 719-730 (2011).

19. Yang, W. et al. Nuclear PKM2 regulates beta-catenin transactivation upon EGFR activation. Nature 480, 118-122 (2011).

20. Lu, Z. Nonmetabolic functions of pyruvate kinase isoform M2 in controlling cell cycle progression and tumorigenesis. Chinese J Cancer 31, 5-7 (2012).

21. Yang, W. et al. ERK1/2-dependent phosphorylation and nuclear translocation of PKM2 promotes the Warburg effect. Nat. Cell Biol. 14, 1295-1304 (2012).

22. Yang, W. et al. EGFR-induced and PKCepsilon monoubiquitylation-dependent NF-kappaB activation upregulates PKM2 expression and promotes tumorigenesis. Mol. Cell 48, 771-784 (2012).

23. Yang, W. et al. PKM2 phosphorylates histone $\mathrm{H} 3$ and promotes gene transcription and tumorigenesis. Cell 150, 685-696 (2012).

24. Lu, Z. PKM2 functions as a histone kinase. Cell Cycle 11, 4101-4102 (2012).

25. Harris, I., McCracken, S. \& Mak, T. W. PKM2: a gatekeeper between growth and survival. Cell Res. 22, 447-449 (2012).

26. Jiang, Y. et al. PKM2 Regulates Chromosome Segregation and Mitosis Progression of Tumor Cells. Mol. Cell 53, 75-87 (2013).

27. Kuan, C. T., Wikstrand, C. J. \& Bigner, D. D. EGF mutant receptor vIII as a molecular target in cancer therapy. Endocr. Relat. Cancer 8, 83-96 (2001).

28. Potapova, T. A. et al. The reversibility of mitotic exit in vertebrate cells. Nature 440, 954-958 (2006).

29. Bohnert, K. A. \& Gould, K. L. On the cutting edge: post-translational modifications in cytokinesis. Trends Cell Biol. 21, 283-292 (2011).

30. Christofk, H. R., Vander Heiden, M. G., Wu, N., Asara, J. M. \& Cantley, L. C. Pyruvate kinase M2 is a phosphotyrosine-binding protein. Nature 452, 181-186 (2008).

31. Gao, X., Wang, H., Yang, J. J., Liu, X. \& Liu, Z. R. Pyruvate kinase M2 regulates gene transcription by acting as a protein kinase. Mol. Cell 45, 598-609 (2012).

32. Lu, Z. \& Hunter, T. Ubiquitylation and proteasomal degradation of the p21(Cip1), p27(Kip1) and p57(Kip2) CDK inhibitors. Cell Cycle 9, 2342-2352 (2010).

33. Sanbe, A. et al. Abnormal cardiac structure and function in mice expressing nonphosphorylatable cardiac regulatory myosin light chain 2. J. Biol. Chem. 274, 21085-21094 (1999).

34. Scruggs, S. B. et al. Ablation of ventricular myosin regulatory light chain phosphorylation in mice causes cardiac dysfunction in situ and affects neighboring myofilament protein phosphorylation. J. Biol. Chem. 284, 5097-5106 (2009).

35. Thaiparambil, J. T., Eggers, C. M. \& Marcus, A. I. AMPK regulates mitotic spindle orientation through phosphorylation of myosin regulatory light chain Mol. Cell Biol. 32, 3203-3217 (2012).

36. Banko, M. R. et al. Chemical genetic screen for AMPKalpha2 substrates uncovers a network of proteins involved in mitosis. Mol. Cell 44, 878-892 (2011).

37. Matsumura, F., Yamakita, Y. \& Yamashiro, S. Myosin light chain kinases and phosphatase in mitosis and cytokinesis. Arch. Biochem. Biophys. 510, 76-82 (2011). 
38. Riento, K. \& Ridley, A. J. Rocks: multifunctional kinases in cell behaviour. Nat. Rev. Mol. Cell Biol. 4, 446-456 (2003).

39. Lowery, D. M. et al. Proteomic screen defines the Polo-box domain interactome and identifies Rock2 as a Plk1 substrate. EMBO J. 26, 2262-2273 (2007).

40. Anastasiou, D. et al. Inhibition of pyruvate kinase M2 by reactive oxygen species contributes to cellular antioxidant responses. Science 334, 1278-1283 (2011).

41. Craig, R. \& Woodhead, J. L. Structure and function of myosin filaments. Curr. Opin. Struct. Biol. 16, 204-212 (2006).

42. Goto, H. et al. Aurora-B regulates the cleavage furrow-specific vimentin phosphorylation in the cytokinetic process. J. Biol. Chem. 278, 8526-8530 (2003).

43. Israelsen, W. J. et al. PKM2 isoform-specific deletion reveals a differential requirement for pyruvate kinase in tumor cells. Cell 155, 397-409 (2013).

44. Wang, Y. H. et al. Cell-state-specific metabolic dependency in hematopoiesis and leukemogenesis. Cell 158, 1309-1323 (2014).

45. Tani, K., Singer-Sam, J., Munns, M. \& Yoshida, A. Molecular cloning and structure of an autosomal processed gene for human phosphoglycerate kinase. Gene 35, 11-18 (1985).

46. Yaffe, M. B. et al. Sequence-specific and phosphorylation-dependent proline isomerization: a potential mitotic regulatory mechanism. Science 278, 1957-1960 (1997).

47. Mazurek, S. Pyruvate kinase type M2: a key regulator within the tumour metabolome and a tool for metabolic profiling of tumours. Ernst. Schering. Found. Symp. Proc. 4, 99-124 (2007).

48. Dombrauckas, J. D., Santarsiero, B. D. \& Mesecar, A. D. Structural basis for tumor pyruvate kinase M2 allosteric regulation and catalysis. Biochemistry 44, 9417-9429 (2005)

49. Mazurek, S., Boschek, C. B., Hugo, F. \& Eigenbrodt, E. Pyruvate kinase type M2 and its role in tumor growth and spreading. Semin. Cancer. Biol. 15, 300-308 (2005).

50. Cortes-Cros, M. et al. M2 isoform of pyruvate kinase is dispensable for tumor maintenance and growth. Proc. Natl Acad. Sci. USA 110, 489-494 (2013).

51. Taus, T. et al. Universal and confident phosphorylation site localization using phosphoRS. J. Proteome Res. 10, 5354-5362 (2011).

52. Lu, Z. et al. Activation of protein kinase C triggers its ubiquitination and degradation. Mol. Cell Biol. 18, 839-845 (1998).

53. Fang, D. et al. Phosphorylation of beta-catenin by AKT promotes beta-catenin transcriptional activity. J. Biol. Chem. 282, 11221-11229 (2007).
54. Mattoo, R. L. \& Waygood, E. B. An enzymatic method for [32P]phosphoenolpyruvate synthesis. Anal. Biochem. 128, 245-249 (1983).

55. Vander Heiden, M. G. et al. Evidence for an alternative glycolytic pathway in rapidly proliferating cells. Science 329, 1492-1499 (2010).

56. Xia, Y. et al. c-Jun downregulation by HDAC3-dependent transcriptional repression promotes osmotic stress-induced cell apoptosis. Mol. Cell 25, 219-232 (2007).

\section{Acknowledgements}

We thank Lewis Cantley and Costas Lyssiotis for their insightful suggestions for this manuscript. We thank Don Cleveland (Ludwig Institute for Cancer Research, University of California-San Diego, CA) for supplying the plasmids that expressed MRLC1, Bih-Fang Pan (MD Anderson Proteomics Facility) for technical assistance and Ann Sutton for her critical reading of this manuscript. This work was supported by National Cancer Institute grants 2R01 CA109035 (Z.L.), 1R0 CA169603 (Z.L.), and CA16672 (Cancer Center Support Grant); James S. McDonnell Foundation 21st Century Science Initiative in Brain Cancer Research Award 220020318 (Z.L.); and the Odyssey Fellowship from MD Anderson (J.Y.).

\section{Author contributions}

This study was conceived by Z.L.; Z.L. and Y.J. designed the study; Y.J., Y.W., T. W., D.H., Y.Z. and X.L. performed the experiments; Q.Z., E.B., S.M., D.L. and S.H. provided reagents and conceptual advice; and Z.L. wrote the paper, with comments from all authors.

\section{Additional information}

Supplementary Information accompanies this paper at http://www.nature.com/ naturecommunications

Competing financial interests: The authors declare no competing financial interests.

Reprints and permission information is available online at http://npg.nature.com/ reprintsandpermissions/

How to cite this article: Jiang, Y. et al. PKM2 phosphorylates MLC2 and regulates cytokinesis of tumour cells. Nat. Commun. 5:5566 doi: 10.1038/ncomms6566 (2014) 